\title{
COLLOCATION METHODS FOR INDEX 1 DAES WITH A SINGULARITY OF THE FIRST KIND
}

\author{
OTHMAR KOCH, ROSWITHA MÄRZ, DIRK PRAETORIUS, AND EWA WEINMÜLLER
}

\begin{abstract}
We study the convergence behavior of collocation schemes applied to approximate solutions of BVPs in linear index 1 DAEs which exhibit a critical point at the left boundary. Such a critical point of the DAE causes a singularity within the inherent ODE system. We focus our attention on the case when the inherent ODE system is singular with a singularity of the first kind, apply polynomial collocation to the original DAE system and consider different choices of the collocation points such as equidistant, Gaussian or Radau points. We show that for a well-posed boundary value problem for DAEs having a sufficiently smooth solution, the global error of the collocation scheme converges with the order $O\left(h^{s}\right)$, where $s$ is the number of collocation points. Superconvergence cannot be expected in general due to the singularity, not even for the differential components of the solution. The theoretical results are illustrated by numerical experiments.
\end{abstract}

\section{INTRODUCTION}

In recent years, a lot of effort has been put into the analysis and numerical treatment of BVPs in ODEs which can exhibit singularities. Such problems are typically given in the following form:

$$
\begin{aligned}
& t^{\alpha} z^{\prime}(t)=M(t) z(t)+f(t, z(t)), \quad t \in(0,1], \\
& g(z(0), z(1))=0, \quad z \in C[0,1],
\end{aligned}
$$

where $\alpha \geq 1, z$ is an $n$-dimensional real function, $M$ is a smooth $n \times n$ matrix, and $f \in \mathbb{R}^{n}$ and $g \in \mathbb{R}^{p}$ are smooth functions. For $\alpha=1$ the problem is called singular with a singularity of the first kind; for $\alpha>1$ it is essentially singular (singularity of the second kind). The search for efficient numerical methods to solve (1a) is strongly motivated by numerous applications from physics [9, 10, 23, 42, chemistry [13, 39, 41], mechanics [12], ecology [30, 37], or economics [14, 15, 24]. Also, research activities in related fields, such as the computation of connecting orbits in dynamical systems 38, or singular Sturm-Liouville problems [6, benefit from techniques developed for problems of the form (1a). In this paper we will extend the techniques developed in the context of ODEs to DAEs.

Our first objective was to provide a sound theoretical basis and the implementation of an open domain MATLAB code for the numerical solution of BVPs with a singularity of the first kind, $\alpha=1$. To compute the numerical solution of (1a), we use collocation at points in the interior of a collocation interval. Our decision to use collocation was motivated by its advantageous convergence properties for (1a).

Received by the editor April 29, 2008 and, in revised form, March 4, 2009.

2000 Mathematics Subject Classification. Primary 65L80; Secondary 65L70. 
The convergence order is at least equal to the stage order of the method, while in the presence of a singularity, other high-order methods show order reductions and become inefficient (see for example 22]); cf. [5, 21, 25]. The above convergence results mean that a collocation scheme with $s$ inner collocation points constitutes a high-order basic solver $\left(O\left(h^{s}\right)\right.$ uniformly in $\left.t\right)$, robust with respect to the singularity of the first kind. Here, we denote by $h$ the maximal stepsize in a (nonequidistant) grid.

In order to solve the ODE systems efficiently, the meshes have to be adapted to the solution behavior. For singular problems, we aim at meshes which are not affected by the steep direction field, staying coarse also close to the singularity when the solution is smooth in that region. To design a mesh adaptation procedure, we need an efficient asymptotically correct a posteriori estimate for the error of the numerical solution. Such a global error estimate was introduced in [4] and is based on the defect correction principle. We could show that for a collocation method of order $O\left(h^{s}\right)$, the error of the estimate (the difference between the exact global error and its estimate) is of order $O\left(h^{s+1}\right)$; see [5, 25. This asymptotically correct error estimate yields a reliable basis for an efficient mesh selection procedure. Our grid adaptation procedure results in grids which adequately reflect the solution behavior.

The final step was to implement the above algorithm and to provide an open domain MATLAB code sbvp1.0 for nonlinear problems with an error estimation routine and a grid selection strategy; see 2, 3. Due to its advantageous convergence properties, collocation is the most robust and dependable solver for singular ODEs; cf. [3, 21. Consequently, we expected collocation to have similar properties in the context of singular DAEs and we will be able to confirm this hypothesis in this article.

In the past and also more recently, several authors successfully applied collocation to well-posed BVPs in index 1 DAEs with no singularities. In [11, 31, nonlinear systems of DAEs with constant kernel of $G_{x^{\prime}}$ have been studied and superconvergence results for Gaussian and Lobatto points were given. The first attempts to provide respective software go back to 1994; see [1]. In the scope of the collocation code COLDAE are semi-explicit problems. Collocation methods applied to solve linear and nonlinear BVPs in index 1 DAEs (without singularities) have been recently analyzed in 32, 33, respectively. Here, the system is assumed to be given in a form of separated sets of equations involving derivatives and derivative free equations. Collocation at Gaussian points and Lobatto points is used to treat those separated equation subsets, respectively. According to our experience, in case of singularities, we need to restrict ourselves to Gaussian points, because the use of Radau or Lobatto points often results in an ill-posed discretized system, due to the unboundedness of the associated canonical projector; cf. [28.

Much progress has been made concerning DAE theory and applications, but there are still many questions left open. In particular, the numerical treatment of critical points and singularities is just emerging. With this paper we are giving the first insight into the behavior of polynomial collocation in the context of singular linear index 1 DAE systems.

Only a few years ago, the concept of DAEs with properly stated leading term was introduced and studied; cf. 7, 16, 34. In contrast to the widely used DAE formulation $E(t) x^{\prime}(t)+F(t) x(t)=g(t)$, this concept aims at a proper and natural 
description of the involved solution derivatives. In particular, one considers linear DAEs written in the form

$$
A(t)(D(t) x(t))^{\prime}+B(t) x(t)=g(t), \quad t \in[a, b],
$$

with continuous coefficient matrices $A(t) \in \mathbb{R}^{m \times n}, D(t) \in \mathbb{R}^{n \times m}, B(t) \in \mathbb{R}^{m \times m}$, where $A(t)$ and $D(t)$ are assumed to be well-matched in the sense that $\operatorname{ker} A(t) \oplus$ $\mathcal{R}(D(t))=\mathbb{R}^{n}$. One of the advantages of this precise description of the problem structure is that there exists an inherent explicit regular ODE (IERODE) uniquely determined by the problem data; see [16, 17, 18. Under mild assumptions, DAEs in standard form can be reformulated to have properly stated leading terms. For DAEs with properly stated leading terms arising in applications, see [16]. Linear BVPs for regular DAEs with tractability index 1 are investigated in detail in [8].

In [36, linear index 1 DAEs with properly stated leading term and type $1 A$ critical points have been analyzed. This means that after decoupling the system using the matrix chain technique developed in [7] into the differential and algebraic components, the related inherent ODE exhibits a singularity of the first or second kind.

In [28, our experimental results showing the convergence of collocation schemes applied to solve (2) have been collected, and in [27, a convergence analysis for the above problem subject to initial conditions has been given. It turns out that for appropriately smooth problem data in (2) the stage order is retained. This means that the global error of the collocation scheme is at least $O\left(h^{s}\right)$ uniformly in $t$. In this article, we will formulate the respective convergence results for BVPs in index 1 DAEs with a singularity of the first kind.

\section{Convergence of COllocation methods}

2.1. Problem specification. We investigate the convergence of collocation for index 1 DAEs, where the so-called inherent $O D E$ may have a singularity of the first kind. In this section we analyze the error of collocation methods applied to a linear system of DAEs given in the following form:

$$
A(t)(D(t) x(t))^{\prime}+B(t) x(t)=g(t), \quad t \in(0,1],
$$

where $A(t) \in \mathbb{R}^{m \times n}, D(t) \in \mathbb{R}^{n \times m}, B(t) \in \mathbb{R}^{m \times m}, g(t), x(t) \in \mathbb{R}^{m}$, with $n \leq m$. All data in (3), that is, the matrix functions $A, D$, and $B$, and the function $g$, are assumed to be at least continuous on $[0,1]$. Moreover, we require that

$$
\begin{array}{ll}
\operatorname{ker} A(t)=\{0\}, & t \in(0,1], \\
\mathcal{R}(D(t))=\mathbb{R}^{n}, & t \in[0,1] .
\end{array}
$$

Condition (5) means that the matrix $D(t)$ has full constant rank on the closed interval. The structure (44) and (51) means that the system (3) has a properly stated leading term on $(0,1]$; cf. [35]. In order to describe the boundary conditions which are necessary and sufficient for (3) to be well-posed, we decouple this system using techniques from [7]. To this end we define

$$
N_{0}(t):=\operatorname{ker}(A(t) D(t)), \quad t \in(0,1] .
$$

Note that $N_{0}(t)=\operatorname{ker} D(t), t \in[0,1]$. 
Let us denote by $Q_{0}$ a continuous pointwise projector function onto $\operatorname{ker} D$, i.e. $Q_{0}(t)^{2}=Q_{0}(t), \mathcal{R}\left(Q_{0}(t)\right)=\operatorname{ker} D(t), t \in[0,1]$, and let $P_{0}:=I-Q_{0}$. Next define

$$
\begin{aligned}
& G_{0}(t):=A(t) D(t), \quad t \in[0,1], \\
& G_{1}(t):=G_{0}(t)+B(t) Q_{0}(t), \quad t \in[0,1] .
\end{aligned}
$$

In the following, we discuss systems (3) which are regular with tractability index 1 on the interval $(0,1]$, which means that the matrix $G_{1}(t)$ is nonsingular for $t \in$ $(0,1]$. We recall that the concept of a tractability index is related to properly defined projector functions and a special matrix function sequence characterizing the structure of the DAE; cf. [40].

Finally, we introduce the pointwise generalized inverse $D^{-}$of $D$ uniquely defined by the following requirements:

$$
D^{-} D D^{-}=D^{-}, D D^{-} D=D, D D^{-}=I, D^{-} D=P_{0},
$$

which need to hold pointwise on $[0,1]$. Note that $D^{-}$is also continuous on $[0,1]$.

We want to incorporate the case where the inherent ODE associated with (3) exhibits a singularity of the first kind; see 20.

It was demonstrated in 7 that with the above assumptions the solutions of the DAE (3) can be decoupled on $(0,1]$ into the differential components Dx and the algebraic components $Q_{0} x$. While $u=D x$ satisfies the explicit inherent ODE,

$$
u^{\prime}(t)+D(t) G_{1}^{-1}(t) B(t) D(t)^{-} u(t)=D(t) G_{1}^{-1}(t) g(t), \quad t \in(0,1],
$$

the algebraic components are given by

$$
Q_{0}(t) x(t)=-Q_{0}(t) G_{1}^{-1}(t) B(t) D(t)^{-} u(t)+Q_{0}(t) G_{1}^{-1}(t) g(t)
$$

and the solutions of (3) can be expressed as

$$
x(t)=D(t)^{-} u(t)+Q_{0}(t) x(t), \quad t \in(0,1] .
$$

We are interested in solutions being smooth on the whole interval $[0,1]$ since we intend to apply a high-order collocation scheme for their approximation. However, $G_{1}(t)$ becomes singular for $t=0$, in general. The asymptotic behavior of (10) related to a singularity of the first kind arises when we assume that $G_{1}(0)$ is singular but $t G_{1}^{-1}(t)$ has a continuous extension on $[0,1]$. Consequently, we can rewrite the matrix $D(t) G_{1}^{-1}(t) B(t) D(t)^{-}$and obtain

$$
D(t) G_{1}^{-1}(t) B(t) D(t)^{-}=:-\frac{1}{t} M(t),
$$

where $M \in C[0,1]$. For the subsequent existence and uniqueness analysis we require $M \in C^{1}[0,1]$, which means that the problem data needs to be appropriately smooth. Let us denote the right-hand side of (10) by $f(t)$. Then we arrive at the inherent ODE of the form

$$
u^{\prime}(t)=\frac{1}{t} M(t) u(t)+f(t), \quad t \in(0,1] .
$$

As mentioned before, we are especially interested in smooth solutions $x$ and therefore $u$ needs to be at least in $C[0,1]$. It turns out that the smoothness of $u$ depends on the smoothness of $f$ and the eigenstructure of $M(0)$. The boundary conditions associated with (13) have to be chosen such that a well-posed singular boundary value problem results. The theoretical background for this problem class, where $f \in C[0,1]$, is discussed in detail in [20] and [26], for example. In order to use this standard theory, we assume that $G_{1}^{-1}(t) g(t)$ and thus $f(t)$ are in $C[0,1]$. 
In this paper we focus our attention on boundary value problems for singular ODE systems (13), which can equivalently be expressed as a well-posed initial value problem with initial conditions at $t^{*}=0$ or terminal conditions at $t^{*}=1$. This means a restriction on the spectrum of the matrix $M(0)$ from (12); see [26, 29] for a detailed explanation of this fact. The reason for the above assumption is that we intend to employ a shooting argument in the course of the analysis.

A singular initial value problem posed at $t^{*}=0$ for the differential equation (13) is well-posed if and only if the spectrum of $M(0)$ contains no eigenvalues with positive real parts and the initial value satisfies $u(0)=\gamma \in \operatorname{ker} M(0)$. A singular terminal value problem posed at $t^{*}=1$ is well-posed if and only if the spectrum of $M(0)$ contains no eigenvalues with negative real parts and the invariant subspace associated with the eigenvalue zero coincides with the nullspace of $M(0)$.

The inherent ODE (13) is augmented by the boundary conditions

$$
B_{a} u(0)+B_{b} u(1)=\beta,
$$

with appropriately chosen $n \times n$ matrices $B_{a}, B_{b}$, and right-hand side $\beta$. For the original DAE (3), this yields the boundary conditions

$$
B_{a} D(0) x(0)+B_{b} D(1) x(1)=\beta .
$$

For a well-posed boundary value problem (13)-(14), the continuous solution can be represented in the form

$$
u(t)=E c+t F(t), \quad t \in[0,1],
$$

where the columns of $E$ are a basis of $\operatorname{ker} M(0)$ and $F(t) \in C[0,1]$; cf. 20]. Consequently, the solution of the full problem (3) and (15) is continuous on $[0,1]$, cf. (11), if the limit

$$
\Theta_{c}:=\lim _{t \rightarrow 0^{+}} Q_{0}(t) G_{1}^{-1}(t) B(t) D(t)^{-} E c
$$

exists, and hence we can set

$$
\begin{aligned}
x_{0}:=x(0)= & D(0)^{-} E c-Q_{0}(0) \Theta_{c} \\
& +Q_{0}(0)\left(-\lim _{t \rightarrow 0^{+}} t G_{1}^{-1}(t) B(t) D(t)^{-} F(t)+\lim _{t \rightarrow 0^{+}} G_{1}^{-1}(t) g(t)\right) .
\end{aligned}
$$

In order to ensure that for any continuous solution $u$ of the inherent ODE (13) there exists a continuous solution $x$ of the DAE (3), we additionally assume that

$$
\Theta:=\lim _{t \rightarrow 0^{+}} Q_{0}(t) G_{1}^{-1}(t) B(t) D(t)^{-} R
$$

exists, where $R \in \mathbb{R}^{n \times n}$ is a projector onto ker $M(0)$. Consequently, we have $\Theta_{c}=\Theta E c$, and

$$
\begin{aligned}
x_{0}= & D(0)^{-} E c-Q_{0}(0) \Theta E c \\
& +Q_{0}(0)\left(-\lim _{t \rightarrow 0^{+}} t G_{1}^{-1}(t) B(t) D(t)^{-} F(t)+\lim _{t \rightarrow 0^{+}} G_{1}^{-1}(t) g(t)\right) .
\end{aligned}
$$

It is important to note that in order to be able to set up a well-posed analytical problem, three (sufficient) conditions have been imposed: We have assumed that

$$
t G_{1}^{-1}(t), \quad G_{1}^{-1}(t) g(t), \quad Q_{0}(t) G_{1}^{-1}(t) B(t) D(t)^{-} E
$$


have continuous extensions on $[0,1]$. The last assumption can also be expressed in terms of the so-called canonical projector,

$$
Q_{\text {can }}(t):=Q_{0}(t) G_{1}^{-1}(t) B(t) .
$$

In general, $Q_{c a n}(t)$ has to be continuous on $[0,1]$ to guarantee (19).

Remark. We exemplify the meaning of assumptions (19) by considering a special DAE system given by

$$
\begin{aligned}
t x_{1}^{\prime}(t)+B_{11}(t) x_{1}(t)+B_{12}(t) x_{2}(t) & =g_{1}(t), \\
B_{21}(t) x_{1}(t)+B_{22}(t) x_{2}(t) & =g_{2}(t),
\end{aligned}
$$

where $B_{11}(t), B_{12}(t), B_{21}(t), B_{22}(t) \in \mathbb{R}^{m \times m}, g_{1}(t), g_{2}(t) \in \mathbb{R}^{m}$, and $x(t)=$ $\left(x_{1}(t), x_{2}(t)\right)^{T} \in \mathbb{R}^{2 m}$. It follows immediately that we can write the above system in the form (3), where

$$
A(t)=\left(\begin{array}{c}
t I \\
0
\end{array}\right), \quad D(t)=\left(\begin{array}{ll}
I & 0
\end{array}\right), \quad B(t)=\left(\begin{array}{cc}
B_{11}(t) & B_{12}(t) \\
B_{21}(t) & B_{22}(t)
\end{array}\right) .
$$

Let

$$
Q_{0}(t):=\left(\begin{array}{cc}
0 & 0 \\
0 & I
\end{array}\right)
$$

Then

$$
G_{1}(t)=\left(\begin{array}{cc}
t I & B_{12}(t) \\
0 & B_{22}(t)
\end{array}\right), \quad G_{1}^{-1}(t)=\left(\begin{array}{cc}
\frac{1}{t} I & -\frac{1}{t} B_{12}(t) B_{22}^{-1}(t) \\
0 & B_{22}^{-1}(t)
\end{array}\right)
$$

and

$$
Q_{0}(t) G_{1}^{-1}(t) B(t) D(t)^{-}=\left(\begin{array}{c}
0 \\
B_{22}^{-1}(t) B_{21}(t)
\end{array}\right) .
$$

Let us assume that $B_{22}(t)$ is nonsingular on $[0,1]$. Then we can decouple (21a), and (3) is an index $1 \mathrm{DAE}$ on $(0,1]$. Moreover, $t G_{1}^{-1}(t)$ and $Q_{0}(t) G_{1}^{-1}(t) B(t) D(t)^{-} R$ are continuous on $[0,1]$. The data of the corresponding inherent ODE (13) read:

$$
M(t)=B_{11}(t)-B_{12}(t) B_{22}^{-1}(t) B_{21}(t), \quad f(t)=\frac{1}{t}\left(g_{1}(t)-B_{12}(t) B_{22}^{-1}(t) g_{2}(t)\right),
$$

where $M(t), f(t)=G_{1}^{-1}(t) g(t) \in C[0,1]$. Clearly, the differential component is $u(t)=D(t) x(t)=x_{1}(t)$, and the algebraic component is

$$
Q_{0}(t) x(t)=\left(\begin{array}{c}
0 \\
x_{2}(t)
\end{array}\right), \quad x_{2}(t)=-B_{22}^{-1}(t) B_{21}(t) u(t)+B_{22}^{-1}(t) g_{2}(t) .
$$

If we only assume that $B_{22}(t)$ is nonsingular on $(0,1]$ and $B_{22}(0)$ is singular, then it is clear that we will require additional conditions to guarantee that the inherent ODE exhibits a singularity of the first kind, that it has a continuous solution $u$, and that this solution yields a smooth solution $x$. In particular, these requirements are satisfied if the matrices $t B_{22}^{-1}(t), B_{22}^{-1}(t) B_{21}(t)$, and $B_{12}(t) B_{22}^{-1}(t)$ have continuous extensions on the closed interval $[0,1]$.

For a high-order method to work efficiently, it is necessary that the analytical solution $x$ is appropriately smooth. This means that the original problem data need to be smooth enough and, additionally, all positive real parts of the eigenvalues of $M(0)$ need to be sufficiently large; cf. [20]. 
In the next section, we apply polynomial collocation to approximate solutions of (3) by means of an enlarged system,

$$
\begin{aligned}
& A(t) u^{\prime}(t)+B(t) x(t)=g(t), \\
& D(t) x(t)-u(t)=0, \quad t \in(0,1],
\end{aligned}
$$

which can be brought into the standard form

$$
\widehat{A}(t)(\widehat{D}(t) \widehat{x}(t))^{\prime}+\widehat{B}(t) \widehat{x}(t)=\widehat{g}(t), \quad t \in(0,1],
$$

where $\widehat{x}(t)=(x(t), u(t))^{T}, \widehat{g}(t)=(g(t), 0)^{T}$, and

$$
\widehat{A}(t)=\left(\begin{array}{c}
A(t) \\
0
\end{array}\right), \quad \widehat{D}(t)=\left(\begin{array}{ll}
0 & I
\end{array}\right)=: \widehat{D}, \quad \widehat{B}(t)=\left(\begin{array}{cc}
B(t) & 0 \\
D(t) & -I
\end{array}\right) .
$$

Problem (24) is a regular DAE system with properly stated leading term and tractability index 1 on $(0,1]$. To see this, note that $\widehat{D}(t)$ is constant. Therefore, we define the related matrices $\widehat{G}_{0}(t), \widehat{Q}_{0}$, and $\widehat{G}_{1}(t)$ as

$$
\widehat{G}_{0}(t):=\widehat{A}(t) \widehat{D}=\left(\begin{array}{cc}
0 & A(t) \\
0 & 0
\end{array}\right), \quad \widehat{Q}_{0}:=\left(\begin{array}{ll}
I & 0 \\
0 & 0
\end{array}\right)
$$

and

respectively. Moreover,

$$
\widehat{G}_{1}(t):=\widehat{G}_{0}(t)+\widehat{B}(t) \widehat{Q}_{0}=\left(\begin{array}{cc}
B(t) & A(t) \\
D(t) & 0
\end{array}\right)
$$

$$
\operatorname{ker} \widehat{G}_{1}=\left\{z \in \mathbb{R}^{m+n}, z=\left(z_{1}, z_{2}\right)^{T} ; z_{1}=Q_{0} w, z_{2}=D w, w \in \operatorname{ker} G_{1}\right\}
$$

for all $t \in[0,1]$, which means that $\widehat{G}_{1}$ is nonsingular on $(0,1]$ and $\widehat{G}_{1}(0)$ is singular, simultaneously with $G_{1}$. From the standard decoupling procedure applied to (24), the same inherent ODE (13) results, and for the solution $\widehat{x}$ on $(0,1]$ the following representation holds:

$$
\widehat{x}(t)=\left(\begin{array}{l}
x(t) \\
u(t)
\end{array}\right)=\left(\begin{array}{c}
\left(I-Q_{0}(t) G_{1}^{-1}(t) B(t)\right) D(t)^{-} u(t)+Q_{0}(t) G_{1}^{-1}(t) g(t) \\
u(t)
\end{array}\right) .
$$

It is easily seen that the matrices $t \widehat{G}_{1}^{-1}(t)$ and $\widehat{Q}_{0}(t) \widehat{G}_{1}^{-1}(t) \widehat{B}(t) \widehat{D}(t)-\widehat{R}$, where $\widehat{R}=R$, are continuous on $[0,1]$.

2.2. Collocation methods. For the theoretical discussion of collocation methods, we define the meshes

$$
\Delta:=\left\{\tau_{0}, \tau_{1}, \ldots, \tau_{N}\right\}
$$

and $h_{i}:=\tau_{i+1}-\tau_{i}, i=0, \ldots, N-1, \tau_{0}=0, \tau_{N}=1$. For reasons of simplicity, we restrict the discussion to equidistant meshes, $h_{i}=h, i=0, \ldots, N-1$. However, the results also hold for nonuniform meshes, which have a limited variation in the step sizes. For collocation, $s$ distinct points $t_{i, j}:=\tau_{i}+h_{i} \rho_{j}, j=1, \ldots, s$, are inserted in each subinterval $\left(\tau_{i}, \tau_{i+1}\right)$. Since we want to focus on Gaussian points, we restrict ourselves to interior collocation points, where $\rho_{1}>0$ and $\rho_{s}<1$. A grid with equidistant interior collocation points is illustrated in Figure 1.

Now, let us denote by $\mathcal{B}_{s}$ the Banach space of continuous, piecewise polynomial functions $q \in \mathbb{P}_{s}$ of degree $\leq s, s \in \mathbb{N}$, equipped with the maximum norm $\|\cdot\|_{\infty}$. In the following, we denote by $q$ the vector-valued functions from $\mathcal{B}_{s}$ independently of the number of their components. By $p \in \mathcal{B}_{s}$ we denote an approximation to the exact solution $x$ of (3), (15), and by $q \in \mathcal{B}_{s}$ an approximation to the exact solution 


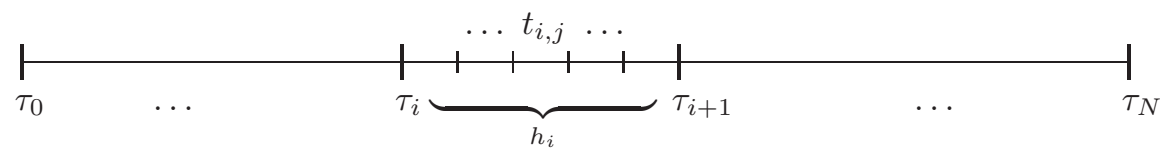

FIGURE 1. The computational grid

$u$ of the inherent ODE (13); cf. (25). The numerical scheme defining $p$ and $q$ has the form

$$
\begin{aligned}
& A\left(t_{i, j}\right) q^{\prime}\left(t_{i, j}\right)+B\left(t_{i, j}\right) p\left(t_{i, j}\right)=g\left(t_{i, j}\right), \\
& D\left(t_{i, j}\right) p\left(t_{i, j}\right)-q\left(t_{i, j}\right)=0, \\
& B_{a} q(0)+B_{b} q(1)=\beta,
\end{aligned}
$$

where $j=1, \ldots, s$ and $i=0, \ldots, N-1$. It is clear by inspection of the number of unknowns and equations that further conditions will be necessary to close the system for the numerical treatment. Clearly, these additional conditions have to be consistent with the original DAEs. Various choices are possible, e.g.,

$$
B(0) p(0)-g(0) \in \lim _{t \rightarrow 0^{+}} \mathcal{R}(A(t)), \quad q(0)=D(0) p(0),
$$

or

$$
B(1) p(1)-g(1) \in \mathcal{R}(A(1)), \quad q(1)=D(1) p(1) .
$$

We first treat the case of boundary value problems which can equivalently be posed as initial value problems at $t^{*}=0$. As we will demonstrate at the end of this section, it is sufficient to investigate the convergence for the IVP case. Consequently, we discuss the solution of

$$
\begin{aligned}
& A(t)(D(t) x(t))^{\prime}+B(t) x(t)=g(t), \\
& D(0) x(0)=\gamma,
\end{aligned}
$$

which is at least continuous on $[0,1]$ and satisfies, see (17),

$$
Q_{0}(0) x(0)=Q_{0}(0) x_{0} .
$$

Recall that in the initial condition (32) the vector $\gamma$ can be freely chosen in ker $M(0)$, and (33) is a consistent initial condition closing the system for the numerical treatment.

When applying collocation to (31)-(33), we are seeking piecewise polynomial functions $p$ and $q$ in $\mathcal{B}_{s}$, which for $j=1, \ldots, s$ and $i=0, \ldots, N-1$ satisfy

$$
\begin{aligned}
& A\left(t_{i, j}\right) q^{\prime}\left(t_{i, j}\right)+B\left(t_{i, j}\right) p\left(t_{i, j}\right)=g\left(t_{i, j}\right), \\
& D\left(t_{i, j}\right) p\left(t_{i, j}\right)-q\left(t_{i, j}\right)=0, \\
& D(0) p(0)=\gamma, \\
& Q_{0}(0) p(0)=Q_{0}(0) x_{0}, \quad P_{0}(0) p(0)=D(0)^{-} q(0) .
\end{aligned}
$$

The following arguments are very similar to those given in the analysis of collocation methods applied to ordinary differential equations with a singularity of the first kind. Thus, to keep the presentation concise we refer to [5, 21, 25] for many of the technical details. First, we study the existence and uniqueness of polynomial functions $p, q \in \mathcal{B}_{s}$ satisfying (34)-(37). As in the analytical case discussed 
in Section 2.1, the system can be decoupled, yielding collocation equations for the inherent ODE $, j=1, \ldots, s, i=0, \ldots, N-1$,

$$
\begin{aligned}
& q^{\prime}\left(t_{i, j}\right)=\frac{1}{t_{i, j}} M\left(t_{i, j}\right) q\left(t_{i, j}\right)+f\left(t_{i, j}\right), \\
& q(0)=\gamma \in \operatorname{ker} M(0),
\end{aligned}
$$

and the value assignments, $j=1, \ldots, s, \quad i=0, \ldots, N-1$,

$$
\begin{aligned}
p\left(t_{i, j}\right)= & \left(I-Q_{0}\left(t_{i, j}\right) G_{1}^{-1}\left(t_{i, j}\right) B\left(t_{i, j}\right)\right) D\left(t_{i, j}\right)^{-} q\left(t_{i, j}\right) \\
& \quad+Q_{0}\left(t_{i, j}\right) G_{1}^{-1}\left(t_{i, j}\right) g\left(t_{i, j}\right), \\
p(0)= & D(0)^{-} \gamma+Q_{0}(0) x_{0} .
\end{aligned}
$$

The results in [5, 21, 25] show that the collocation equations (38), (39) have a unique solution which satisfies

$$
q(t)=E c+t \varphi(t)=\gamma+t \varphi(t), \quad \varphi \in \mathcal{B}_{s} ;
$$

cf. (16). Conditions (40) and (41) uniquely define a piecewise polynomial $p \in \mathcal{B}_{s}$.

2.3. Error analysis: Initial value problems at $t^{*}=0$. We now analyze the error of the approximation $p$ provided by (34)-(37). Let us introduce an error function $\widehat{e} \in \mathcal{B}_{s}$ defined by

$$
\begin{aligned}
\widehat{e}^{\prime}\left(t_{i, j}\right) & =\widehat{x}^{\prime}\left(t_{i, j}\right)-\widehat{p}^{\prime}\left(t_{i, j}\right), \quad j=1, \ldots, s, i=0, \ldots, N-1, \\
\widehat{e}(0) & =0,
\end{aligned}
$$

cf. [5, 21, where $\widehat{x}(t)=(x(t), u(t))^{T}$ is the exact solution, and $\widehat{p}(t)=(p(t), q(t))^{T}$ is its approximation by a collocation polynomial. Moreover, $\widehat{e}(t):=\left(e(t), e_{u}(t)\right)^{T}$. Trivially,

$$
\widehat{e}^{\prime}(t)=\sum_{\mu=1}^{s} L_{\mu}\left(\frac{t-\tau_{i}}{h}\right) \widehat{x}^{\prime}\left(t_{i, \mu}\right)-\widehat{p}^{\prime}(t), \quad t \in\left(\tau_{i}, \tau_{i+1}\right),
$$

where $L_{\mu}$ denotes the $\mu$ th Lagrange polynomial (of degree $s-1$ ) associated with the abscissae $\rho_{1}, \ldots, \rho_{s}$ on the interval $[0,1]$. In our analysis we wish to take into account that possibly the differential and the algebraic solution components have different smoothness. Thus, we assume that

$$
u=D x \in C^{\tilde{k}+1}[0,1], \quad x \in C^{\tilde{\ell}+1}[0,1],
$$

and set

$$
k:=\min \{\tilde{k}, s\}, \quad \ell:=\min \{\tilde{\ell}, s\} .
$$

From standard results for interpolation, see [19], we can conclude that

$$
\widehat{e}^{\prime}(t)=\widehat{x}^{\prime}(t)-\widehat{p}^{\prime}(t)+\left(\begin{array}{c}
O\left(h^{\ell}\right) \\
O\left(h^{k}\right)
\end{array}\right) .
$$

Due to (44), integration of (46) yields

$$
\widehat{e}(t)=\widehat{x}(t)-\widehat{p}(t)+t\left(\begin{array}{c}
r(t) \\
s(t)
\end{array}\right)
$$


with $r(t)=O\left(h^{\ell}\right)$ and $s(t)=O\left(h^{k}\right)$. Thus, $\widehat{e}$ satisfies the collocation scheme

$$
\begin{aligned}
& A\left(t_{i, j}\right) e_{u}^{\prime}\left(t_{i, j}\right)+B\left(t_{i, j}\right) e\left(t_{i, j}\right)=t_{i, j} B\left(t_{i, j}\right) r\left(t_{i, j}\right), \\
& D\left(t_{i, j}\right) e\left(t_{i, j}\right)-e_{u}\left(t_{i, j}\right)=t_{i, j}\left(D\left(t_{i, j}\right) r\left(t_{i, j}\right)-s\left(t_{i, j}\right)\right), \\
& e(0)=0, \quad e_{u}(0)=0 .
\end{aligned}
$$

Again, we can reduce the problem to the collocation scheme applied to the inherent ODE related to (48)-(49), cf. (38), and obtain

$$
\begin{aligned}
& e_{u}^{\prime}\left(t_{i, j}\right)=\frac{1}{t_{i, j}} M\left(t_{i, j}\right) e_{u}\left(t_{i, j}\right)-M\left(t_{i, j}\right) O\left(h^{k}\right), \\
& e_{u}(0)=0 .
\end{aligned}
$$

To prove this statement we multiply (48) by $D\left(t_{i, j}\right) G_{1}^{-1}\left(t_{i, j}\right)$ and obtain

$$
\begin{aligned}
& e_{u}^{\prime}\left(t_{i, j}\right)+D\left(t_{i, j}\right) G_{1}^{-1}\left(t_{i, j}\right) B\left(t_{i, j}\right) D\left(t_{i, j}\right)^{-} D\left(t_{i, j}\right) e\left(t_{i, j}\right) \\
& \quad=t_{i, j} D\left(t_{i, j}\right) G_{1}^{-1}\left(t_{i, j}\right) B\left(t_{i, j}\right) r\left(t_{i, j}\right),
\end{aligned}
$$

taking into account the relations $D G_{1}^{-1} A=I, D G_{1}^{-1} B Q_{0}=0$, and $D G_{1}^{-1} B=$ $D G_{1}^{-1} B D^{-} D$. We now express $D\left(t_{i, j}\right) e\left(t_{i, j}\right)$ using (49), as

$$
D\left(t_{i, j}\right) e\left(t_{i, j}\right)=e_{u}\left(t_{i, j}\right)+t_{i, j} D\left(t_{i, j}\right) r\left(t_{i, j}\right)-t_{i, j} s\left(t_{i, j}\right),
$$

and insert this into (53). Rearranging yields

$$
\begin{aligned}
& e_{u}^{\prime}\left(t_{i, j}\right)-\frac{1}{t_{i, j}} M\left(t_{i, j}\right) e_{u}\left(t_{i, j}\right) \\
& \quad=-M\left(t_{i, j}\right) s\left(t_{i, j}\right)+\left(M\left(t_{i, j}\right) D\left(t_{i, j}\right)+t_{i, j} D\left(t_{i, j}\right) G_{1}^{-1}\left(t_{i, j}\right) B\left(t_{i, j}\right)\right) r\left(t_{i, j}\right) .
\end{aligned}
$$

The second term on the right-hand side vanishes, since

$$
\begin{aligned}
M D+t D G_{1}^{-1} B & =-t D G_{1}^{-1} B D^{-} D+t D G_{1}^{-1} B \\
& =t D G_{1}^{-1} B\left(I-D^{-} D\right)=t D G_{1}^{-1} B Q_{0}=0
\end{aligned}
$$

and this completes the argument.

Note that the inhomogeneity in (51) remains uniformly bounded for $t \in[0,1]$. Therefore, we can use [5, 21], see also (42), to conclude that

$$
e_{u}(t)=t O\left(h^{k}\right)
$$

Consequently, the error function $e \in \mathcal{B}_{s}$ can be uniquely described by assigning its values,

$$
\begin{aligned}
e\left(t_{i, j}\right)= & \left(I-Q_{0}\left(t_{i, j}\right) G_{1}^{-1}\left(t_{i, j}\right) B\left(t_{i, j}\right)\right) D\left(t_{i, j}\right)^{-} e_{u}\left(t_{i, j}\right) \\
& \quad+Q_{0}\left(t_{i, j}\right) G_{1}^{-1}\left(t_{i, j}\right) t_{i, j} B\left(t_{i, j}\right) r\left(t_{i, j}\right), \\
e(0)= & 0 .
\end{aligned}
$$

Finally, we have $e\left(t_{i, j}\right)=O\left(h^{\min \{\ell, k\}}\right)$ and since $e \in \mathcal{B}_{s}$, it immediately follows that $e(t)=O\left(h^{\min \{\ell, k\}}\right)$ and

$$
x(t)-p(t)=O\left(h^{\min \{\ell, k\}}\right)
$$

hold. 
2.4. Error analysis: Terminal value problems at $t^{*}=1$. Consider the case where the boundary value problem can equivalently be posed as a terminal value problem at $t^{*}=1$. The convergence proof given above requires a few modifications in that case. The collocation equations (34)-(35) are now augmented by the terminal conditions, cf. (11), (25),

$$
\begin{aligned}
D(1) p(1) & =\beta, \\
Q_{0}(1) p(1) & =-Q_{0}(1) G_{1}^{-1}(1) B(1) D(1)^{-} \beta+Q_{0}(1) G_{1}^{-1}(1) g(1), \\
P_{0}(1) p(1) & =D(1)^{-} q(1) .
\end{aligned}
$$

Clearly, (44) is replaced by $\widehat{e}(1)=0$, whence (47) becomes

$$
\widehat{e}(t)=\widehat{x}(t)-\widehat{p}(t)+(1-t)\left(\begin{array}{c}
r(t) \\
s(t)
\end{array}\right) .
$$

Consequently, the error function satisfies the collocation scheme

$$
\begin{aligned}
& A\left(t_{i, j}\right) e_{u}^{\prime}\left(t_{i, j}\right)+B\left(t_{i, j}\right) e\left(t_{i, j}\right)=\left(1-t_{i, j}\right) B\left(t_{i, j}\right) r\left(t_{i, j}\right), \\
& D\left(t_{i, j}\right) e\left(t_{i, j}\right)-e_{u}\left(t_{i, j}\right)=\left(1-t_{i, j}\right) D\left(t_{i, j}\right) r\left(t_{i, j}\right)-\left(1-t_{i, j}\right) s\left(t_{i, j}\right), \\
& e(1)=0, \quad e_{u}(1)=0 .
\end{aligned}
$$

The collocation scheme for the associated inherent ODE reads

$$
\begin{aligned}
e_{u}^{\prime}\left(t_{i, j}\right) & =\frac{1}{t_{i, j}} M\left(t_{i, j}\right) e_{u}\left(t_{i, j}\right)-\frac{1}{t_{i, j}} M\left(t_{i, j}\right)\left(1-t_{i, j}\right) O\left(h^{k}\right), \\
e_{u}(1) & =0 .
\end{aligned}
$$

This yields, see 25] for the proof,

$$
e_{u}(t)=O\left(h^{k}\right) .
$$

Moreover,

$$
\begin{aligned}
e\left(t_{i, j}\right)= & \left(I-Q_{0}\left(t_{i, j}\right) G_{1}^{-1}\left(t_{i, j}\right) B\left(t_{i, j}\right)\right) D\left(t_{i, j}\right)^{-} e_{u}\left(t_{i, j}\right) \\
& \quad+\left(1-t_{i, j}\right) Q_{0}\left(t_{i, j}\right) G_{1}^{-1}\left(t_{i, j}\right) B\left(t_{i, j}\right) r\left(t_{i, j}\right), \\
e(1)= & 0 .
\end{aligned}
$$

According to assumptions (19), this yields $e\left(t_{i, j}\right)=t_{i, j}^{-1} O\left(h^{\min \{\ell, k\}}\right)$ and therefore $e(t)=O\left(h^{\min \{\ell, k\}-1}\right)$; i.e., we observe an order reduction if compared with (58). The error bound $e\left(t_{i, j}\right)=O\left(h^{\min \{\ell, k\}}\right)$ from (58), however, follows under the additional assumption that the canonical projector

$$
Q_{c a n}(t):=Q_{0}(t) G_{1}^{-1}(t) B(t)
$$

has a continuous extension on $[0,1]$.

2.5. Error analysis: Boundary value problems. To conclude the error analysis, we give the shooting argument which demonstrates that the analysis of initial value problems given above is sufficient for the analysis of boundary value problems for the DAE (3). This argument is the same as the one given in [5, 21] for boundary value problems of singular ODEs, and carries over to our present analysis. Recall that $t G_{1}^{-1}(t), G_{1}^{-1}(t) g(t)$, and $Q_{0}(t) G_{1}^{-1}(t) B(t) D(t)^{-} E$ are assumed to have continuous extensions on $[0,1]$. The columns of $E$ form a basis for ker $M(0)$. 
The solution of the inherent ODE (13) can be written as

$$
u(t)=\sum_{\mu=1}^{r} a_{\mu} u_{\mu}(t)+\tilde{u}(t),
$$

where $U=\left(u_{\mu}\right)_{\mu=1, \ldots, r}$ is the solution of the homogeneous matrix ODE with initial condition $U(0)=E$, and the particular solution $\tilde{u}$ satisfies the inhomogeneous equation, albeit with homogeneous initial condition. It is easy to see from Section 2.1 that this implies a representation of $x$ as

$$
x(t)=\sum_{\mu=1}^{r} a_{\mu} x_{\mu}(t)+\tilde{x}(t),
$$

where $X=\left(x_{\mu}\right)_{\mu=1, \ldots, r}$ with

$$
x_{\mu}(t)=\left(I-Q_{0}(t) G_{1}^{-1}(t) B(t)\right) D(t)^{-} u_{\mu}(t)
$$

solves the matrix DAE

$$
\begin{aligned}
& A(t)(D(t) X(t))^{\prime}+B(t) X(t)=0, \\
& D(0) X(0)=E,
\end{aligned}
$$

and $\tilde{x}=\left(I-Q_{0} G_{1}^{-1} B\right) D^{-} \tilde{u}+Q_{0} G_{1}^{-1} g$ satisfies

$$
\begin{aligned}
& A(t)(D(t) \tilde{x}(t))^{\prime}+B(t) \tilde{x}(t)=g(t), \\
& D(0) \tilde{x}(0)=0 .
\end{aligned}
$$

Due to our assumptions, $X$ and $\tilde{x}$ are continuous on $[0,1]$. An analogous representation holds for the collocation solution $p$,

$$
p(t)=\sum_{\mu=1}^{r} b_{\mu} p_{\mu}(t)+\tilde{p}(t),
$$

where $P=\left(p_{\mu}\right)_{\mu=1, \ldots, r}$ and $\tilde{p}$ are the collocation solutions of the problems (71) $-(172)$ and (73)-(74), respectively. Similarly as in the ODE case, see [5, 21], substitution of the solution representations (69) and (75) into (14) yields

$$
a_{\mu}-b_{\mu}=O\left(h^{k}\right)
$$

provided that the matrix $B_{a} E+B_{b} P(1)$ is nonsingular. Consequently, the convergence result

$$
\|u-q\|_{\infty}=O\left(h^{k}\right), \quad\|x-p\|_{\infty}=O\left(h^{\min \{\ell, k\}}\right)
$$

holds also for the solution of the boundary value problem (3), (15).

The considerations for problems posed at $t^{*}=1$ are analogous.

\section{NumeriCAL EXPERIMENTS}

In this section, we illustrate the convergence behavior of the quantities

$$
\begin{array}{ll}
\operatorname{gex}_{\mathrm{tau}}:=\|p-x\|_{\mathrm{tau}}, & \operatorname{gex}_{\mathrm{tcol}}:=\|p-x\|_{\mathrm{tcol}}, \\
\text { geu }_{\mathrm{tau}}:=\|q-u\|_{\mathrm{tau}}, & g_{\mathrm{tcol}}:=\|q-u\|_{\mathrm{tcol}},
\end{array}
$$

numerically. The first two expressions are the maximal values of the global errors of the solution $x$ in the mesh points only (tau) 1 and in all grid points, including

\footnotetext{
${ }^{1}$ Cf. the definition of $\Delta$ in Section 2.2
} 
$\tau_{i}$ and collocation points $t_{i, j},(\mathrm{tcol})$, respectively. The last two expressions are the respective global errors for the differential solution components $u$.

Before continuing with the results of our numerical experiments, we stress that in the above theory the important assumptions (19),

$$
t G_{1}^{-1}(t) \in C[0,1], \quad G_{1}^{-1}(t) g(t) \in C[0,1], \quad Q_{0}(t) G_{1}^{-1}(t) B(t) D(t)^{-} E \in C[0,1]
$$

have been made. They are sufficient for the stage order $s$ of the collocation scheme to hold. Let $x$ and $u$ denote the solutions of the DAE and the inherent ODE, respectively. Provided that $u$ is sufficiently smooth, (76) yields, for equidistant interior collocation points,

$$
\begin{array}{ll}
\text { geu }_{\text {tau }}=O\left(h^{s+1}\right), & \text { geu } \\
\text { geu }_{\text {tau }}=O\left(h^{s}\right), & \text { geu } \\
\text { tcol } & =O\left(h^{s+1}\right),
\end{array}
$$

for $s$ odd or even, respectively; see [28. Moreover, note that for Gaussian collocation points the superconvergence behavior $O\left(h^{2 s}\right)$ in $\Delta$ does not hold in general, a well-known fact in the context of singular ODEs. Rather, the same orders as in (77a) are observed (uniform superconvergence).

If the inherent ODE is an initial value problem and if $x$ is sufficiently smooth, theory predicts the same order of convergence for gex $x_{\mathrm{tau}}$ and $g e x_{\mathrm{tcol}}$ as for $u$; cf. Section 2.3. If the inherent ODE is a terminal value or boundary value problem, our analysis additionally requires

$$
Q_{\text {can }}(t):=Q_{0}(t) G_{1}^{-1}(t) B(t) \in C[0,1]
$$

to ensure this optimal convergence behavior; cf. Section 2.4. In this case, theory predicts that

$$
\text { gex }_{\mathrm{tau}}=O\left(h^{s}\right), \quad \text { gex } \mathrm{tcol}=O\left(h^{s}\right) .
$$

If the assumptions (76) and (78) are violated, order reductions in the algebraic components might occur. In particular, order reductions can be due to the behavior of the canonical projector $Q_{c a n}(t)$ for $t \rightarrow 0^{+}$, in the case when $Q_{c a n}$ becomes unbounded in this limit. In the following, we consider two problem classes and try to illustrate important aspects of the theory by appropriately constructed model problems; cf. Tables 1 and 2 for a summary. Therein, we highlight order reductions in the sense that the stage order is not observed for the numerical experiment, although the exact solutions are sufficiently smooth.

All experiments have been carried out in MATLAB 7 by our new version of sbvp [3, 4.

3.1. Problem class 1 - setting and analytical properties. The system of DAEs in this problem class has the form

$$
\left(\begin{array}{c}
t^{k} \\
1
\end{array}\right)\left(x_{1}(t)+\alpha(t) x_{2}(t)\right)^{\prime}+\left(\begin{array}{cc}
t^{k} \beta(t) & -t^{k} \alpha^{\prime}(t) \\
0 & \gamma(t)-\alpha^{\prime}(t)
\end{array}\right) x(t)=\left(\begin{array}{l}
t^{k} g_{1}(t) \\
g_{2}(t)
\end{array}\right)=: g(t),
$$

where $k \geq 0$. Model problems can be constructed in such a way that the inherent differential equation is regular or singular, with singularity of the first or of the second kind; see 28, for comprehensive experimental results on the convergence of collocation schemes applied to solve such problems.

For the models considered here, $k=0$ and the above system has the form

$$
\left(\begin{array}{l}
1 \\
1
\end{array}\right)\left(x_{1}(t)+\alpha(t) x_{2}(t)\right)^{\prime}+\left(\begin{array}{cc}
\beta(t) & -\alpha^{\prime}(t) \\
0 & \gamma(t)-\alpha^{\prime}(t)
\end{array}\right) x(t)=: g(t) .
$$


TABLE 1. Overview of numerical experiments, where the inherent ODEs are classified as initial (IVP), terminal (TVP), or boundary value problem (BVP). We stress that the exact solutions for all but the final Problem 2.4 are smooth. Order reductions are indicated in the fifth column

\begin{tabular}{|c|c|c|c|c|c|}
\hline Experiment & Assumptions (76) & $Q_{c a n}$, cf. (78) & ODE & Order & Reference \\
\hline Prbl. 1.1 & satisfied & $\overline{\bar{O} O(1 / t)}$ & $\overline{\mathrm{IVP}}$ & stage & $\begin{array}{lll}28 & \text { Prbl. 1.22] } \\
\end{array}$ \\
\hline Prbl. 1.2 & not satisfied & $O\left(1 / t^{2}\right)$ & TVP & reduction & \begin{tabular}{|l|l}
28 & Prbl. 1.21] \\
\end{tabular} \\
\hline Prbl. 2.1 & satisfied & continuous & BVP & stage & \begin{tabular}{|ll}
28 & Prbl. 4.2]
\end{tabular} \\
\hline Prbl. 2.2 & satisfied & continuous & BVP & stage & \begin{tabular}{|l|l} 
28 & Prbl. 4.3]
\end{tabular} \\
\hline Prbl. 2.3 & satisfied & $O(1 / t)$ & TVP & stage & \begin{tabular}{|l|l}
28 & Prbl. 4.5] \\
\end{tabular} \\
\hline Prbl. 2.4 & satisfied & $O(1 / t)$ & TVP & reduction & [28, Prbl. 4.6] \\
\hline
\end{tabular}

TABLE 2. Experimentally observed convergence rates for different collocation schemes with $s=3,4$; cf. 28] for details. Order reductions are highlighted in italic.

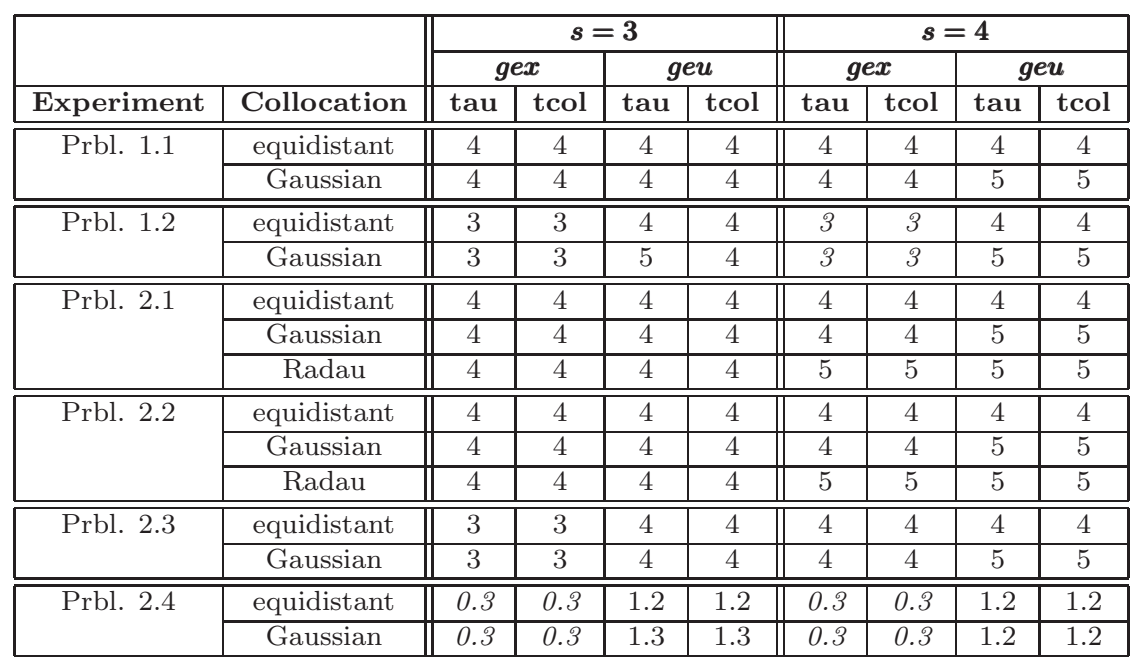

In order to specify $x$ we need to calculate the canonical projector (20),

$$
\text { (81) } Q_{\text {can }}(t)=Q_{0}(t) G_{1}^{-1}(t) B(t)=\frac{1}{\alpha(t) \beta(t)+\gamma(t)}\left(\begin{array}{cc}
\alpha(t) \beta(t) & -\alpha(t) \gamma(t) \\
-\beta(t) & \gamma(t)
\end{array}\right) \text {. }
$$

Moreover, with

we obtain

$$
Q_{0}(t) G_{1}^{-1}(t)=\frac{1}{\alpha(t) \beta(t)+\gamma(t)}\left(\begin{array}{cc}
\alpha(t) & -\alpha(t) \\
-1 & 1
\end{array}\right)
$$

$$
P_{\text {can }}(t)=\frac{1}{\alpha(t) \beta(t)+\gamma(t)}\left(\begin{array}{cc}
\gamma(t) & \alpha(t) \gamma(t) \\
\beta(t) & \alpha(t) \beta(t)
\end{array}\right)
$$

and finally, the explicit formula for the solution $x$ of the DAE (80) follows from

$$
x(t)=\frac{1}{\alpha(t) \beta(t)+\gamma(t)}\left(\begin{array}{c}
\gamma(t) \\
\beta(t)
\end{array}\right) u(t)+\frac{1}{\alpha(t) \beta(t)+\gamma(t)}\left(\begin{array}{c}
\alpha(t) g_{1}(t)-\alpha(t) g_{2}(t) \\
-g_{1}(t)+g_{2}(t)
\end{array}\right) .
$$


It is important to note that the sign of the term

$$
-\frac{\beta(t)\left(\gamma(t)-\alpha^{\prime}(t)\right)}{\varphi(t)}
$$

cf. (10), with $\beta(t)\left(\gamma(t)-\alpha^{\prime}(t)\right)$ evaluated at $t=0$, is crucial and decides the boundary conditions necessary for the solution $u$ (and $x$ ) to be at least in $C[0,1]$; cf. 20] for the analysis of singular ODEs.

Problem 3.1. We choose $\alpha(t)=-1$, whence $\varphi(t)=\gamma(t)-\beta(t)$. With $\gamma(t)=t+2$ and $\beta(t)=2$, we obtain $\varphi(t)=t$ and the equation for $u$ reads:

$$
u^{\prime}(t)=-\frac{2(t+2)}{t} u(t)+\frac{1}{t}\left((t+2) g_{1}(t)-2 g_{2}(t)\right) .
$$

The requirement $\varphi(t) \neq 0$ for $t \in(0,1]$ is satisfied because $\gamma(t)-\beta(t)=t \neq 0$ for $t \in(0,1]$. We additionally choose $u(t)=t e^{5 t}$. The right-hand side $g$ is specified below, and the solution of the problem has the form $x(t)=\left(-(6 t+1) e^{5 t} / 2,-(8 t+\right.$ 1) $\left.e^{5 t} / 2\right)^{T}$. Finally, we arrive at the system of DAEs

$$
\left(\begin{array}{l}
1 \\
1
\end{array}\right)\left(x_{1}(t)-x_{2}(t)\right)^{\prime}+\left(\begin{array}{cc}
2 & 0 \\
0 & t+2
\end{array}\right) x(t)=\left(\begin{array}{c}
-t e^{5 t} \\
-\frac{8 t+7}{2} t e^{5 t}
\end{array}\right),
$$

subject to the boundary conditions $x_{1}(0)-x_{2}(0)=0,2 x_{1}(1)-3 x_{2}(1)=6.5 e^{5}$. Concerning the boundary conditions, we note that here

$$
\frac{1}{t} M(t)=-\frac{2(t+2)}{t}, \quad M(0)=-4,
$$

and therefore we need to prescribe the value $u(0)=x_{1}(0)-x_{2}(0)=0$. The second boundary condition is equivalent to $\beta(t) x_{1}(t)-\gamma(t) x_{2}(t)=g_{1}(t)-g_{2}(t)$; cf. (30). For this example, condition (76) is satisfied. From $\alpha(t)=-1, \gamma(t)=t+2, \beta(t)=$ $2, \alpha(t) \beta(t)+\gamma(t)=t$, we obtain

$$
\begin{aligned}
G_{1}^{-1}(t) & =\frac{1}{\alpha(t) \beta(t)+\gamma(t)}\left(\begin{array}{cc}
\alpha(t)+\gamma(t)-\alpha^{\prime}(t) & -\alpha(t)+\alpha(t) \beta(t)+\alpha^{\prime}(t) \\
-1
\end{array}\right) \\
& =\frac{1}{t}\left(\begin{array}{cc}
t+1 & -1 \\
-1 & 1
\end{array}\right) .
\end{aligned}
$$

This means that

$$
G_{1}^{-1}(t)=O\left(\frac{1}{t}\right) \Rightarrow t G_{1}^{-1}(t)=O(1)
$$

Moreover

$$
\begin{aligned}
G_{1}^{-1}(t) g(t) & =\frac{1}{t}\left(\begin{array}{cc}
t+1 & -1 \\
-1 & 1
\end{array}\right)\left(\begin{array}{c}
-t e^{5 t} \\
-\frac{8 t+7}{2} t e^{5 t}
\end{array}\right) \\
& =\left(\begin{array}{cc}
t+1 & -1 \\
-1 & 1
\end{array}\right)\left(\begin{array}{c}
-e^{5 t} \\
-\frac{8 t+7}{2} e^{5 t}
\end{array}\right) \in C[0,1]
\end{aligned}
$$

and $E=0$. The associated canonical projector (20) is

$$
Q_{c a n}(t)=\left(\begin{array}{cc}
-\frac{2}{t} & \frac{2+t}{t} \\
-\frac{2}{t} & \frac{2+t}{t}
\end{array}\right)=O\left(\frac{1}{t}\right) .
$$

Since the solution $x$ is appropriately smooth, in Table 3 we observe the following convergence behavior for $s=4$ equidistant collocation points,

$$
g^{e} x_{\mathrm{tau}}=g_{\mathrm{e}} x_{\mathrm{tcol}}=\operatorname{geu}_{\mathrm{tau}}=g e u_{\mathrm{tcol}}=O\left(h^{s}\right)=O\left(h^{4}\right),
$$


TABle 3. Convergence results for Problem 1.1: $s=4$, equidistant collocation points.

\begin{tabular}{|c|c||c|c|c||c|c|c|}
\hline \multicolumn{2}{|c||}{ Uniform Mesh } & \multicolumn{3}{|c||}{$\boldsymbol{g e x}_{\text {tau }}$} & \multicolumn{3}{c|}{$\boldsymbol{g e x}_{\text {tcol }}$} \\
\hline $\mathbf{N}$ & $\mathbf{h}$ & error & order & const. & error & order & const. \\
\hline \hline 20 & $5.00 e-02$ & $2.321 e-03$ & 4.0 & $3.548 e+02$ & $2.321 e-03$ & 4.0 & $3.548 e+02$ \\
40 & $2.50 e-02$ & $1.459 e-04$ & 4.0 & $3.627 e+02$ & $1.459 e-04$ & 4.0 & $3.627 e+02$ \\
80 & $1.25 e-02$ & $9.155 e-06$ & 4.0 & $3.652 e+02$ & $9.155 e-06$ & 4.0 & $3.652 e+02$ \\
160 & $6.25 e-03$ & $5.744 e-07$ & 4.0 & $3.659 e+02$ & $5.744 e-07$ & 4.0 & $3.659 e+02$ \\
\hline \hline \multicolumn{2}{|c||}{ Uniform Mesh } & \multicolumn{3}{|c|}{$\boldsymbol{g e u _ { \text { tau } }}$} \\
\hline $\mathbf{N}$ & $\mathbf{h}$ & error & order & const. & error & order & const. \\
\hline \hline 20 & $5.00 e-02$ & $1.032 e-04$ & 4.0 & $1.742 e+01$ & $1.032 e-04$ & 4.0 & $1.742 e+01$ \\
40 & $2.50 e-02$ & $6.429 e-06$ & 4.0 & $1.674 e+01$ & $6.429 e-06$ & 4.0 & $1.674 e+01$ \\
80 & $1.25 e-02$ & $4.015 e-07$ & 4.0 & $1.653 e+01$ & $4.015 e-07$ & 4.0 & $1.653 e+01$ \\
160 & $6.25 e-03$ & $2.509 e-08$ & 4.0 & $1.646 e+01$ & $2.509 e-08$ & 4.0 & $1.646 e+01$ \\
\hline
\end{tabular}

TABle 4. Convergence results for Problem 1.1: $s=4$, Gaussian collocation points.

\begin{tabular}{|c|c|c|c|c|c|c|c|}
\hline \multicolumn{2}{|c|}{ Uniform Mesh } & \multicolumn{3}{|c|}{$g e x_{\text {tau }}$} & \multicolumn{3}{|c|}{$g e x_{\mathrm{tcol}}$} \\
\hline $\mathbf{N}$ & $\mathbf{h}$ & error & order & const. & error & order & const. \\
\hline 20 & $5.00 e-02$ & $8.633 e-04$ & 4.0 & $1.319 e+02$ & $8.633 e-04$ & 4.0 & $1.319 e+02$ \\
\hline 40 & $2.50 e-02$ & $5.426 e-05$ & 4.0 & $1.349 e+02$ & $5.426 e-05$ & 4.0 & $1.349 e+02$ \\
\hline 80 & $1.25 e-02$ & $3.406 e-06$ & 4.0 & $1.358 e+02$ & $3.406 e-06$ & 4.0 & $1.358 e+02$ \\
\hline 160 & $6.25 e-03$ & $2.137 e-07$ & 4.0 & $1.361 e+02$ & $2.137 e-07$ & 4.0 & $1.361 e+02$ \\
\hline \multicolumn{2}{|c|}{ "Uniform Mesh } & \multicolumn{3}{|c|}{$\overline{g e u_{\text {tau }}}$} & \multicolumn{3}{|c|}{$\overline{g e u_{\text {tcol }}}$} \\
\hline $\mathbf{N}$ & $\mathbf{h}$ & error & order & const. & error & order & const. \\
\hline 20 & $\overline{5.00 e-02}$ & $2.479 e-09$ & $\overline{5.5}$ & $3.804 e-02$ & $6.356 e-06$ & 4.8 & $\overline{1.243 e+01}$ \\
\hline 40 & $2.50 e-02$ & $6.377 e-11$ & 5.3 & $1.839 e-02$ & $2.100 e-07$ & 4.9 & $1.597 e+01$ \\
\hline 80 & $1.25 e-02$ & $1.801 e-12$ & 5.1 & $1.120 e-02$ & $6.749 e-09$ & 5.0 & $1.856 e+01$ \\
\hline 160 & $6.25 e-03$ & $5.684 e-14$ & 5.0 & $5.533 e-03$ & $2.139 e-10$ & 5.0 & $2.020 e+01$ \\
\hline
\end{tabular}

which is predicted by theory. For $s=4$ Gaussian collocation points, we observe

$$
g^{e} x_{\mathrm{tau}}=g_{e x} x_{\mathrm{tcol}}=O\left(h^{s}\right)=O\left(h^{4}\right), \quad g^{e} u_{\mathrm{tau}}=g e u_{\mathrm{tcol}}=O\left(h^{s+1}\right)=O\left(h^{5}\right) ;
$$

cf. Table 4 Note that although the canonical projector $Q_{\text {can }}(t)=O(1 / t)$ is unbounded for $t \rightarrow 0^{+}$, no order reduction is observed. This is due to the fact that the involved inherent ODE is an initial value problem, and therefore an additional factor $t$ helps to balance the unboundedness of $Q_{\text {can }}(t)=O(1 / t)$; see (55)-(56).

Problem 3.2. Functions $\alpha(t)$ and $\varphi(t)$ are as in Problem 1.1. With $\gamma(t)=\sin t$ and $\beta(t)=t$, we obtain $\varphi(t)=\sin t-t$ and the equation for $u$ reads:

$$
u^{\prime}(t)=-\frac{t \sin t}{\sin t-t} u(t)+\frac{1}{\sin t-t}\left(\sin t g_{1}(t)-t g_{2}(t)\right) .
$$

The requirement $\varphi(t) \neq 0$ for $t \in(0,1]$ is satisfied. We set $u(t)=t-\sin t$, $g_{1}(t)=g_{2}(t)$ are given below, and the solution of the system is $x(t)=(-\sin t,-t)^{T}$. The related system of DAEs is

$$
\left(\begin{array}{l}
1 \\
1
\end{array}\right)\left(x_{1}(t)-x_{2}(t)\right)^{\prime}+\left(\begin{array}{cc}
t & 0 \\
0 & \sin t
\end{array}\right) x(t)=\left(\begin{array}{c}
1-\cos t-t \sin t \\
1-\cos t-t \sin t
\end{array}\right),
$$

boundary conditions are given by $x_{1}(1)-x_{2}(1)=-\sin (1)+1, x_{2}(1)=-1$, and it is clear that this problem must be treated as a terminal value problem from right $(t=1)$ to left $(t=0)$. Since

$$
\lim _{t \rightarrow 0^{+}}\left(-\frac{t \sin t}{\sin t-t}\right)=\lim _{t \rightarrow 0^{+}}\left(\frac{6}{t}\right)=\lim _{t \rightarrow 0^{+}}\left(\frac{\lambda}{t}\right), \lambda>0,
$$


TABle 5. Convergence results for Problem 1.2: $s=2$, equidistant collocation points. One observes order reductions for both components of $x=\left(x_{1}, x_{2}\right)$.

\begin{tabular}{|c|c|c|c|c|c|c|c|}
\hline \multicolumn{2}{|c|}{ Uniform Mesh } & \multicolumn{3}{|c|}{ gex $1_{\text {tau }}$} & \multicolumn{3}{|c|}{ gex $1_{\text {tcol }}$} \\
\hline $\mathbf{N}$ & $\mathbf{h}$ & error & order & const. & error & order & const. \\
\hline$\overline{20}$ & $5.00 e-02$ & $3.165 e-02$ & 1.0 & $\overline{5.587 e-01}$ & $3.165 e-02$ & $\overline{1.0}$ & $\overline{5.587 e-01}$ \\
\hline 40 & $2.50 e-02$ & $1.604 e-02$ & 1.0 & $5.975 e-01$ & $1.604 e-02$ & 1.0 & $5.975 e-01$ \\
\hline 80 & $1.25 e-02$ & $8.072 e-03$ & 1.0 & $6.200 e-01$ & $8.072 e-03$ & 1.0 & $6.200 e-01$ \\
\hline 160 & $6.25 e-03$ & $4.049 e-03$ & 1.0 & $6.330 e-01$ & $4.049 e-03$ & 1.0 & $6.330 e-01$ \\
\hline \multicolumn{2}{|c|}{ Uniform Mesh } & \multicolumn{3}{|c|}{ gex 2 tau } & \multicolumn{3}{|c|}{$\overline{g e x 2_{\text {tcol }}}$} \\
\hline $\mathbf{N}$ & $\mathbf{h}$ & error & order & const. & error & order & const. \\
\hline$\overline{20}$ & $\overline{5.00 e-02}$ & $3.165 e-02$ & $\overline{1.0}$ & $\overline{5.575 e-01}$ & $3.165 e-02$ & 1.0 & $5.575 e-01$ \\
\hline 40 & $2.50 e-02$ & $1.604 e-02$ & 1.0 & $5.971 e-01$ & $1.604 e-02$ & 1.0 & $5.971 e-01$ \\
\hline 80 & $1.25 e-02$ & $8.072 e-03$ & 1.0 & $6.199 e-01$ & $8.072 e-03$ & 1.0 & $6.199 e-01$ \\
\hline 160 & $6.25 e-03$ & $4.049 e-03$ & 1.0 & $6.330 e-01$ & $4.049 e-03$ & 1.0 & $6.330 e-01$ \\
\hline \multicolumn{2}{|c|}{ "Uniform Mesh } & \multicolumn{3}{|c|}{$g e u_{\text {tau }}$} & \multicolumn{3}{|c|}{$\overline{\text { geu }_{\mathrm{tcol}}}$} \\
\hline $\mathbf{N}$ & $\mathbf{h}$ & error & order & const. & error & order & const. \\
\hline 20 & $5.00 e-02$ & $6.260 e-06$ & 2.5 & $1.247 e-02$ & $6.691 e-06$ & 2.4 & $9.998 e-03$ \\
\hline 40 & $2.50 e-02$ & $1.538 e-06$ & 2.0 & $2.697 e-03$ & $1.605 e-06$ & 2.1 & $3.197 e-03$ \\
\hline 80 & $1.25 e-02$ & $3.829 e-07$ & 2.0 & $2.519 e-03$ & $3.920 e-07$ & 2.0 & $2.911 e-03$ \\
\hline 160 & $6.25 e-03$ & $9.563 e-08$ & 2.0 & $2.466 e-03$ & $9.681 e-08$ & 2.0 & $2.712 e-03$ \\
\hline
\end{tabular}

we prescribe the value of $u$ at $t=1, u(1)=x_{1}(1)-x_{2}(1)$. The second boundary condition is again the consistency condition (30).

The conditions (76) and (78) are not satisfied. From $\alpha(t)=-1, \gamma(t)=\sin t$, $\beta(t)=t, \alpha(t) \beta(t)+\gamma(t)=\sin t-t$, it follows that

$$
G_{1}^{-1}(t)=\frac{1}{\sin t-t}\left(\begin{array}{cc}
\sin t-1 & 1-t \\
-1 & 1
\end{array}\right)=O\left(\frac{1}{t^{3}}\right) \Rightarrow t G_{1}^{-1}(t)=O\left(\frac{1}{t^{2}}\right)
$$

However,

$$
\begin{aligned}
G_{1}^{-1}(t) g(t) & =\frac{1}{\sin t-t}\left(\begin{array}{cc}
\sin t-1 & 1-t \\
-1 & 1
\end{array}\right)\left(\begin{array}{l}
1-\cos t-t \sin t \\
1-\cos t-t \sin t
\end{array}\right) \\
& =\left(\begin{array}{c}
1-\cos t-t \sin t \\
0
\end{array}\right)
\end{aligned}
$$

and $E=0$. The canonical projector (20) has the form

$$
Q_{\text {can }}(t)=\left(\begin{array}{cc}
-\frac{t}{\sin t-t} & \frac{\sin t}{\sin t-t} \\
-\frac{t}{\sin t-t} & \frac{\sin t}{\sin t-t}
\end{array}\right)=O\left(\frac{1}{t^{2}}\right) .
$$

Due to (82)- (83), we observe clear order reductions; see Tables 5 and 6 . Here, we are dealing with a terminal value problem and there are no additional $t$ factors to balance the behavior of $Q_{\text {can }}(t)$; see (65)- (66). Note that for singular boundary value problems in ODEs, the uniform superconvergence order $O\left(h^{s+1}\right)$ for Gaussian collocation nodes holds in general at both the mesh points $\tau_{i}$ and the collocation points $t_{i j}$.

\subsection{Problem class 2.}

3.2.1. Problems with bounded canonical projector, $B_{22}$ nonsingular. The problems considered in the previous section exhibited scalar inherent ODEs with $M(0) \in \mathbb{R}$. Therefore, the associated BVP could be posed either as an initial value problem, $M(0)<0$, or as a terminal value problem, $M(0)>0$; see Problem 1.1 and Problem 
TABle 6. Convergence results for Problem 1.2: $s=2$, Gaussian collocation points. One observes order reductions for both components of $x=\left(x_{1}, x_{2}\right)$.

\begin{tabular}{|c|c|c|c|c|c|c|c|}
\hline \multicolumn{2}{|c|}{ Uniform Mesh } & \multicolumn{3}{|c|}{$g e x 1_{\text {tau }}$} & \multicolumn{3}{|c|}{ gex $1_{\text {tcol }}$} \\
\hline $\mathrm{N}$ & h & error & order & const. & error & order & const. \\
\hline$\overline{20}$ & $5.00 e-02$ & $3.994 e-02$ & $\overline{1.0}$ & $\overline{77.413 e-01}$ & $3.994 e-02$ & 1.0 & $\overline{7.413 e-01}$ \\
\hline 40 & $2.50 e-02$ & $2.013 e-02$ & 1.0 & $7.716 e-01$ & $2.013 e-02$ & 1.0 & $7.716 e-01$ \\
\hline 80 & $1.25 e-02$ & $1.010 e-02$ & 1.0 & $7.889 e-01$ & $1.010 e-02$ & 1.0 & $7.889 e-01$ \\
\hline 160 & $6.25 e-03$ & $5.062 e-03$ & 1.0 & $7.988 e-01$ & $5.062 e-03$ & 1.0 & $7.988 e-01$ \\
\hline \multicolumn{2}{|c|}{ Uniform Mesh } & \multicolumn{3}{|c|}{ gex $2_{\text {tau }}$} & \multicolumn{3}{|c|}{ gex $2_{\text {tcol }}$} \\
\hline $\mathbf{N}$ & h & error & order & const. & error & order & const. \\
\hline$\overline{20}$ & $5.00 e-02$ & 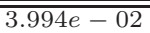 & $\overline{1.0}$ & $\overline{77.405 e-01}$ & $3.994 e-02$ & 1.0 & $\overline{7.405 e-01}$ \\
\hline 40 & $2.50 e-02$ & $2.013 e-02$ & 1.0 & $7.713 e-01$ & $2.013 e-02$ & 1.0 & $7.713 e-01$ \\
\hline 80 & $1.25 e-02$ & $1.010 e-02$ & 1.0 & $7.888 e-01$ & $1.010 e-02$ & 1.0 & $7.888 e-01$ \\
\hline 160 & $6.25 e-03$ & $5.062 e-03$ & 1.0 & $7.987 e-01$ & $5.062 e-03$ & 1.0 & $7.987 e-01$ \\
\hline \multicolumn{2}{|c|}{ "Uniform Mesh } & \multicolumn{3}{|c|}{$\overline{\overline{g e u_{\text {tau }}}}$} & \multicolumn{3}{|c|}{$\overline{g e u_{\text {tcol }}}$} \\
\hline $\mathbf{N}$ & $\mathbf{h}$ & error & order & const. & error & order & const. \\
\hline 20 & $\overline{5.00 e-02}$ & 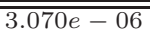 & $\overline{\overline{3.0}}$ & 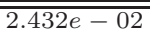 & $3.070 e-06$ & $\overline{\overline{3.0}}$ & $\overline{2.432 e-02}$ \\
\hline 40 & $2.50 e-02$ & $3.840 e-07$ & 3.0 & $2.450 e-02$ & $3.840 e-07$ & 3.0 & $2.450 e-02$ \\
\hline 80 & $1.25 e-02$ & $4.801 e-08$ & 3.0 & $2.456 e-02$ & $4.801 e-08$ & 3.0 & $2.456 e-02$ \\
\hline 160 & $6.25 e-03$ & $6.001 e-09$ & 3.0 & $2.457 e-02$ & $6.001 e-09$ & 3.0 & $2.457 e-02$ \\
\hline
\end{tabular}

1.2 , respectively. In order to study the influence of other eigenstructures of the matrix $M(0)$, we now consider the following higher-dimensional semi-explicit problem posed on the interval $(0,1]$ :

$$
\left(\begin{array}{c}
t I \\
0
\end{array}\right)\left(\left(\begin{array}{ll}
I & 0
\end{array}\right)\left(\begin{array}{l}
x_{1}(t) \\
x_{2}(t)
\end{array}\right)\right)^{\prime}+\left(\begin{array}{ll}
B_{11} & B_{12} \\
B_{21} & B_{22}
\end{array}\right)\left(\begin{array}{l}
x_{1}(t) \\
x_{2}(t)
\end{array}\right)=\left(\begin{array}{l}
g_{1}(t) \\
g_{2}(t)
\end{array}\right)
$$

or explicitly,

$$
\begin{aligned}
t x_{1}^{\prime}(t)+B_{11} x_{1}(t)+B_{12} x_{2}(t) & =g_{1}(t), \\
B_{21} x_{1}(t)+B_{22} x_{2}(t) & =g_{2}(t),
\end{aligned}
$$

where $B_{11}, B_{12}, B_{21}, B_{22} \in \mathbb{R}^{2 \times 2}$ are constant matrices, $g_{1}(t), g_{2}(t) \in C[0,1]$ and the matrix $B_{22}$ is nonsingular. Due to the problem structure we can immediately rewrite (85) to decouple the inherent ODE system and the system of algebraic constraints.

We first express $x_{2}(t)$ from (85b), $x_{2}(t)=B_{22}^{-1}\left(g_{2}(t)-B_{21} x_{1}(t)\right)$, and rewrite (85a) as

$$
x_{1}^{\prime}(t)=-\frac{1}{t}\left(B_{11} x_{1}(t)-B_{12} B_{22}^{-1} B_{21} x_{1}(t)\right)+\frac{1}{t}\left(g_{1}(t)-B_{12} B_{22}^{-1} g_{2}(t)\right) .
$$

Consequently, the inherent ODE system is singular with a singularity of the first kind and has the form

$$
x_{1}^{\prime}(t)=\frac{1}{t} M x_{1}(t)+f(t), \quad t \in(0,1],
$$

where $M=B_{12} B_{22}^{-1} B_{21}-B_{11}$ and $f(t)=\left(g_{1}(t)-B_{12} B_{22}^{-1} g_{2}(t)\right) / t$. Problems 2.1 and 2.2 given below are specified in such a way that $M$ has a special eigenstructure: Problem 2.1 has eigenvalues $\lambda_{1}=0, \lambda_{2}=5$, and for Problem 2.2, $\lambda_{1}=\lambda_{2}=0$.

The initial conditions which guarantee that for $f \in C[0,1]$ the solution $x_{1}(t)$ of (86) is at least in $C[0,1]$ read $Q x_{1}(0)=0$, where $Q$ is a projection onto the subspace of $\mathbb{R}^{2}$ spanned either by the eigenvector associated with the negative eigenvalue of $M$ or by the principal vector associated with the eigenvalue zero (Problem 2.2). For uniqueness we need to prescribe the value of $P x_{1}$, where $P=$ 
$I-Q$. Clearly, $P=S+R$, where $S$ is a projection onto the subspace of $\mathbb{R}^{2}$ spanned by the eigenvector associated with the positive eigenvalue of $M$ (Problem 2.1). Recall that $R$ is a projection onto the subspace of $\mathbb{R}^{2}$ spanned by the eigenvector associated with the eigenvalue zero (Problems 2.1 and 2.2). According to theory, one needs to prescribe $S x_{1}$ at $t=1$, but $R x_{1}$ can be specified at either $t=0$ or $t=1$. We now give the specification of Problems 2.1 and 2.2; for details see [28].

Problem 3.3. The matrices $B_{i j}$ are

$$
B_{11}=\left(\begin{array}{rr}
9 & 12 \\
-8 & -11
\end{array}\right), \quad B_{12}=\left(\begin{array}{rr}
3 & -1 \\
-2 & 1
\end{array}\right), \quad B_{21}=\left(\begin{array}{ll}
1 & 1 \\
2 & 3
\end{array}\right), \quad B_{22}=\left(\begin{array}{ll}
1 & 0 \\
0 & \frac{1}{2}
\end{array}\right) .
$$

For the system (866) we have $\lambda_{1}=0, \lambda_{2}=5$ and

$$
M=\left(\begin{array}{rr}
-10 & -15 \\
10 & 15
\end{array}\right), e v_{0}=\left(\begin{array}{r}
-1.5 \\
1
\end{array}\right), e v_{5}=\left(\begin{array}{r}
-1 \\
1
\end{array}\right), R=\left(\begin{array}{rr}
3 & 3 \\
-2 & -2
\end{array}\right), S=\left(\begin{array}{rr}
-2 & -3 \\
2 & 3
\end{array}\right) .
$$

The functions $g$ and $f$ are specified in such a way that the solution of the Problem 2.1 reads:

$$
x_{1}=\left(\begin{array}{l}
x_{11} \\
x_{12}
\end{array}\right)=\left(\begin{array}{c}
e^{5 t} \sin (t)-1.5+t^{5} \\
\cos (5 t)-t^{5}
\end{array}\right), \quad x_{2}=\left(\begin{array}{c}
x_{21} \\
x_{22}
\end{array}\right)=\left(\begin{array}{c}
\cos (5 t)-1 \\
e^{5 t} \sin (t)
\end{array}\right) .
$$

Here, $Q=0$ and the solution is continuous. We just need to specify boundary conditions which are necessary for uniqueness. We prescribe $\left(S x_{1}(1)\right)_{2}=2 x_{11}(1)+$ $3 x_{12}(1)=2 e^{5} \sin (1)+3 \cos (5)-4$, and for the second condition we specify $\left(R x_{1}(0)\right)_{1}$, which means $x_{11}(0)+x_{12}(0)=-0.5$. For $x_{2}$, we prescribe the consistency condition $B_{22} x_{2}(t)+B_{21} x_{1}(t)=g_{2}(t)$ at $t=0$,

$$
\left(\begin{array}{cc}
1 & 0 \\
0 & \frac{1}{2}
\end{array}\right) x_{2}(0)+\left(\begin{array}{ll}
1 & 1 \\
2 & 3
\end{array}\right) x_{1}(0)=\left(\begin{array}{c}
-0.5 \\
0
\end{array}\right) \text {. }
$$

Problem 3.4. For this model we define the matrices $B_{i j}$ as

$$
B_{11}=\left(\begin{array}{rr}
-9 & -15 \\
8 & 13
\end{array}\right), \quad B_{12}=\left(\begin{array}{rr}
3 & -1 \\
-2 & 1
\end{array}\right), \quad B_{21}=\left(\begin{array}{ll}
1 & 1 \\
2 & 3
\end{array}\right), \quad B_{22}=\left(\begin{array}{ll}
1 & 0 \\
0 & \frac{1}{3}
\end{array}\right) .
$$

In (86)) we now have $\lambda_{1}=\lambda_{2}=0$ and

$$
M=\left(\begin{array}{rr}
6 & 9 \\
-4 & -6
\end{array}\right), e v_{0}=\left(\begin{array}{r}
-3 \\
2
\end{array}\right), p v_{0}=\left(\begin{array}{r}
1 \\
-1
\end{array}\right), R=\left(\begin{array}{rr}
3 & 3 \\
-2 & -2
\end{array}\right), Q=\left(\begin{array}{rr}
-2 & -3 \\
-2 & -3
\end{array}\right) .
$$

Moreover,

$$
x_{1}=\left(\begin{array}{l}
x_{11} \\
x_{12}
\end{array}\right)=\left(\begin{array}{c}
e^{4 t} \sin (t)-3 \\
\cos (4 t)+1
\end{array}\right), \quad x_{2}=\left(\begin{array}{c}
x_{21} \\
x_{22}
\end{array}\right)=\left(\begin{array}{c}
\cos (4 t)-1 \\
e^{4 t} \sin (t)
\end{array}\right) .
$$

We require $\left(Q x_{1}(0)\right)_{1}=0$, or equivalently, $2 x_{11}(0)+3 x_{12}(0)=0$ for the solution $x_{1}$ to be continuous on $[0,1]$. As a second condition we choose to specify $\left(R x_{1}(1)\right)_{1}$, which means $x_{11}(1)+x_{12}(1)=e^{4} \sin (1)+\cos (4)-2$. The consistency conditions for $x_{2}$ read $B_{22} x_{2}(0)+B_{21} x_{1}(0)=g_{2}(0)$, which means

$$
\left(\begin{array}{ll}
1 & 0 \\
0 & \frac{1}{3}
\end{array}\right) x_{2}(0)+\left(\begin{array}{ll}
1 & 1 \\
2 & 3
\end{array}\right) x_{1}(0)=\left(\begin{array}{r}
-1 \\
0
\end{array}\right) .
$$

We stress that, for Problems 2.1 and 2.2, condition (76) is satisfied due to the Remark on page 286. The numerical results are summarized in Table 2, In 28, we have also considered other eigenstructures of the matrix $M$, and it is worth mentioning that collocation at Gaussian points proved robust for all situations. The experiments for this problem class show convergence rates which do not differ from those given in (77a) and (79). Here, we have also used Radau points as 
collocation points. (Lobatto points cannot be used because of the singularity.) As for Gaussian points, superconvergence does not always hold; cf. Table 2 ,

3.2.2. Problems with unbounded canonical projector, $B_{22}$ singular. Here, we continue the study of the behavior of the canonical projector $Q_{c a n}(t)$. The following examples can be written as a modified system (84) and have the form

$$
\left(\begin{array}{c}
t^{\alpha} I \\
0
\end{array}\right)\left(\left(\begin{array}{ll}
I & 0
\end{array}\right)\left(\begin{array}{l}
x_{1}(t) \\
x_{2}(t)
\end{array}\right)\right)^{\prime}+\left(\begin{array}{ll}
B_{11}(t) & B_{12}(t) \\
B_{21}(t) & B_{22}(t)
\end{array}\right)\left(\begin{array}{l}
x_{1}(t) \\
x_{2}(t)
\end{array}\right)=\left(\begin{array}{l}
g_{1}(t) \\
g_{2}(t)
\end{array}\right) .
$$

Here again,

$$
D=\left(\begin{array}{ll}
I & 0
\end{array}\right), \quad D^{-}=\left(\begin{array}{l}
I \\
0
\end{array}\right), \quad Q_{0}=\left(\begin{array}{ll}
0 & 0 \\
0 & I
\end{array}\right) .
$$

Moreover, $B_{22}(t):=t^{\beta} B_{22}, \beta>0, \alpha+\beta=1$, and $B_{22}$ is nonsingular. In this setting,

$$
G_{1}(t)=\left(\begin{array}{cc}
t^{\alpha} I & B_{12}(t) \\
0 & B_{22}(t)
\end{array}\right), \quad G_{1}^{-1}(t)=\left(\begin{array}{cc}
\frac{1}{t^{\alpha}} I & -\frac{1}{t^{\alpha}} B_{12}(t) B_{22}^{-1} \\
0 & B_{22}^{-1}
\end{array}\right),
$$

which means that $t G_{1}^{-1}$ has a continuous extension to $[0,1]$ in the case where all involved matrices are continuous on $[0,1]$. However, the canonical projector

$$
Q_{\text {can }}(t)=Q_{0} G_{1}^{-1}(t) B(t)=\left(\begin{array}{cc}
0 & 0 \\
\frac{1}{t^{\beta}} B_{22}^{-1} B_{21}(t) & I
\end{array}\right)
$$

is unbounded on $[0,1]$ for $\beta>0$. Moreover,

$$
\begin{aligned}
D G_{1}^{-1}(t) B(t) D^{-} & =\frac{1}{t^{\alpha}} B_{11}(t)-\frac{1}{t} B_{12}(t) B_{22}^{-1} B_{21}(t) \\
& =\frac{1}{t}\left(t^{\beta} B_{11}(t)-B_{12}(t) B_{22}^{-1} B_{21}(t)\right),
\end{aligned}
$$

and hence

$$
M(t)=-t^{\beta} B_{11}(t)+B_{12}(t) B_{22}^{-1} B_{21}(t), \quad M(0)=B_{12}(0) B_{22}^{-1} B_{21}(0) .
$$

For the experiments, we choose the matrices $B_{12}(t), B_{21}(t)$ to be constant and the matrices $B_{22}, B_{21}(t)$ to be nonsingular. Moreover, with the choice $B_{12}=B_{21}^{-1}$ and $B_{22}^{-1}=\operatorname{diag}\left(\lambda_{1}, \lambda_{2}\right), \lambda_{i} \neq 0$, the eigenvalues of $M(0)$ are $\lambda_{1}$ and $\lambda_{2}$. Consequently, since $M(0)$ is nonsingular, $R=0$, and the matrix $Q_{0} G_{1}^{-1}(t) B(t) D^{-} R=0$ has a continuous extension on $[0,1]$.

Problem 3.5. The matrices $B_{i j}$ are

$$
B_{11}=\left(\begin{array}{ll}
0 & 0 \\
0 & 0
\end{array}\right), B_{12}=\left(\begin{array}{rr}
3 & -1 \\
-2 & 1
\end{array}\right), B_{21}=\left(\begin{array}{ll}
1 & 1 \\
2 & 3
\end{array}\right), B_{22}(t)=t^{\beta} B_{22}=t^{\beta}\left(\begin{array}{ll}
1 & 0 \\
0 & \frac{1}{5}
\end{array}\right) \text {. }
$$

Also,

$$
B_{22}^{-1}=\left(\begin{array}{ll}
1 & 0 \\
0 & 5
\end{array}\right), M(0)=B_{12} B_{22}^{-1} B_{21}=\left(\begin{array}{rr}
-7 & -12 \\
8 & 13
\end{array}\right) .
$$

System (87) is subject to the boundary conditions

$$
\begin{aligned}
& x_{1}(1)=\sin (1), \quad x_{2}(1)=e^{1}, \\
& x_{1}(1)+x_{2}(1)+x_{3}(1)=\sin (1)+e^{1}+\cos (1), \\
& 2 x_{1}(1)+3 x_{2}(1)+\frac{1}{5} x_{4}(1)=2 \sin (1)+3 e^{1}+\frac{1}{5} e^{-1} .
\end{aligned}
$$


Since the eigenvalues of $M(0)$ are both positive, we prescribe the values of the differential components $x_{1}(t)$ and $x_{2}(t)$ at $t=1$. The remaining two conditions are consistent boundary conditions for the algebraic components. Note that we solve a terminal value problem which generically shows order reductions when $Q_{\text {can }}(t)$ becomes unbounded for $t \rightarrow 0^{+}$.

The right-hand side $g$ which depends on parameters $\alpha, \beta$, and $\ell \geq \alpha$ has been chosen such that the problem has the solution $x(t)=\left(t \sin (t), t e^{t}, \cos (t), t^{\ell} e^{-t}\right)^{T}$; cf. 28 for details. For the experiment, the parameters have been set as follows: $\alpha=0, \beta=1$, and $\ell=3$.

The numerical results for this example can be found in Table 2 For the case where the differential solution components $u(t)$ are smooth, no order reduction is observed, although the projection matrix $Q_{\text {can }}(t)=O(1 / t)$ is unbounded for $t \rightarrow 0^{+}$.

Problem 3.6. We use the same data as in Problem 2.3 except for the right-hand side $g$ which here depends on parameters $\alpha, \beta, \gamma, \delta$ and $\ell$ such that $\ell \geq \alpha \geq$ $0, \alpha+\beta=1, \beta>0, \delta \geq 1, \gamma \geq 0, \gamma+\alpha \geq 0$.

The boundary conditions for the resulting terminal value problem are given by

$$
\begin{aligned}
& x_{1}(1)=\sin (1), \quad x_{2}(1)=e^{1}, \\
& x_{1}(1)+x_{2}(1)+x_{3}(1)=\sin (1)+e^{1}+\cos (1), \\
& 2 x_{1}(1)+3 x_{2}(1)+\frac{1}{5} x_{4}(1)=2 \sin (1)+3 e^{1}+\frac{1}{5} e^{-1} .
\end{aligned}
$$

Again, since the eigenvalues of $M(0)$ are both positive, we prescribe the values of the differential components $x_{1}(t)$ and $x_{2}(t)$ at $t=1$. The remaining two conditions are consistent boundary conditions for the algebraic components. Note that (766) is again satisfied. The solution now has the form $x(t)=\left(t^{\gamma} \sin (t), t^{\delta} e^{t}, \cos (t), t^{\ell} e^{-t}\right)^{T}$, and the differential components $x_{1}$ and $x_{2}$ may become unsmooth. We set $\alpha=0$ and $\beta=1$, and the remaining parameters are specified as $\ell=\frac{5}{2}, \gamma=\frac{6}{5}, \delta=\frac{5}{2}$.

The experiments for this example are reported in Table 2. Now, we observe order reductions due to the fact that the canonical projector (88) is unbounded for $t \rightarrow 0^{+}(\beta=1)$. One would expect to see the convergence order $O\left(h^{1.2}\right)$ owing to the properties of $x$, especially the differential components. However, one loses approximately one additional power of $h$ in $x$ which can be attributed to the $O(1 / t)$ behavior of $Q_{c a n}(t)$.

\section{Summary}

We investigated the convergence behavior of collocation schemes applied to solve BVPs in linear index 1 DAEs with a singularity of the first kind. We have considered a very general analytical problem setting, linear index 1 DAE systems with properly stated leading term, which were required to be well-posed and have sufficiently smooth solutions. For the discussion of the analytical problem and in the convergence analysis of the collocation scheme, we utilized a theoretical decoupling of the system to derive the explicit inherent ODE and the algebraic constraints. We could show that in the case of initial, terminal, and boundary value problems, the global error of the collocation scheme for $s$ equidistant, Gaussian or Radau 
collocation points is $O\left(h^{s}\right)$ uniformly in $t$. More precisely, for equidistant nodes,

$$
\begin{aligned}
& g e x_{\text {tau }}=O\left(h^{s}\right), \quad g e x_{\text {tcol }}=O\left(h^{s}\right), \quad \text { geu } u_{\text {tau }}=O\left(h^{s+1}\right), \quad g e u_{\mathrm{tcol}}=O\left(h^{s+1}\right), \\
& g_{\text {gex }}=O\left(h^{s}\right), \quad g e x_{\text {tcol }}=O\left(h^{s}\right), \quad g e u_{\text {tau }}=O\left(h^{s}\right), g e u_{\text {tcol }}=O\left(h^{s}\right)
\end{aligned}
$$

hold for $s$ odd and even, respectively. By means of experiments we could illustrate the fact that conditions on the problem data which are sufficient for the analytical problem to be well posed turn out to be necessary for the numerical scheme to have the stage order $s$. The superconvergence order $2 s$ for Gaussian points does not hold in general, due to the singularity in the inherent ODE.

Similar convergence results hold also for systems of DAEs with no singularities in the inherent ODE. For initial, terminal, and boundary value problems with smooth solutions, the above convergence orders hold in the case that condition (178) is satisfied. Otherwise, order reductions in the algebraic components may occur.

Clearly, when the solution of the problem is not sufficiently smooth, corresponding order reductions are observed, in line with classical collocation theory.

For Radau collocation superconvergence, $g e x_{\text {tau }}=O\left(h^{2 s-1}\right)$, geu tau $=O\left(h^{2 s-1}\right)$ is observed, and the uniform convergence behavior is $g e x_{\mathrm{tcol}}=O\left(h^{s+1}\right), g e u_{\mathrm{tcol}}=$ $O\left(h^{s+1}\right)$, for all values of $s$, except for the case $s=1$, corresponding to the backward Euler rule showing the expected linear convergence.

\section{REFERENCES}

[1] U.M. Ascher and R.J. Spiteri. Collocation software for boundary value differential-algebraic equations. SIAM J. Sci. Comput., 4:938-952, 1994. MR.1278008 (95a:65004)

[2] W. Auzinger, G. Kneisl, O. Koch, and E. Weinmüller. A solution routine for singular boundary value problems. Techn. Rep. ANUM Preprint Nr. 1/02, Inst. for Appl. Math. and Numer. Anal., Vienna Univ. of Technology, Austria, 2002. Available at http://www.math.tuwien.ac.at/ ${ }^{\sim}$ inst115/preprints.htm.

[3] W. Auzinger, G. Kneisl, O. Koch, and E. Weinmüller. A collocation code for boundary value problems in ordinary differential equations. Numer. Algorithms, 33:27-39, 2003. MR2005549 (2004k:65114)

[4] W. Auzinger, O. Koch, and E. Weinmüller. Efficient collocation schemes for singular boundary value problems. Numer. Algorithms, 31:5-25, 2002. MR 1950909 (2003k:65070)

[5] W. Auzinger, O. Koch, and E. Weinmüller. Analysis of a new error estimate for collocation methods applied to singular boundary value problems. SIAM J. Numer. Anal., 42(6):23662386, 2005. MR2139397 (2006b:65109)

[6] P. Bailey, W. Everitt, and A. Zettl. Computing eigenvalues of singular Sturm-Liouville problems. Results Math., 20:391-423, 1991. MR1122349 (93a:65102)

[7] K. Balla and R. März. A unified approach to linear differential algebraic equations and their adjoint equations. J. Anal. Appl., 21(3):783-802, 2002. MR.1929432 (2003g:34002)

[8] K. Balla and R. März. Linear boundary value problems for differential algebraic equations. Miskolc Mathematical Notes, 5:3-18, 2004. MR2040972 (2004m:65113)

[9] C.J. Budd and R. Kuske. Localised periodic pattern for the non-symmetric generalized SwiftHohenberg equations. Physica D, 208:73-95, 2005. MR 2167908 (2007d:35141)

[10] C.J. Budd and J.F. Williams. Parabolic Monge-Ampère methods for blow-up problems in several spatial dimensions. J. Phys. A, 39:5425-5463, 2006. MR2220768(2006k:35130)

[11] A. Degenhardt. Collocation for transferable differential-algebraic equations. Technical Report 1992-1, Humboldt University Berlin, 1992.

[12] M. Drmota, R. Scheidl, H. Troger, and E. Weinmüller. On the imperfection sensitivity of complete spherical shells. Comp. Mech., 2:63-74, 1987.

[13] G.B. Froment and K.B. Bischoff. Chemical reactor analysis and design. John Wiley \& Sons Inc., New York, 1990.

[14] S. Golub. Measures of restrictions in inward foreign direct investment in OECD countries. OECD Economics Dept. WP Nr. $35 \%$. 
[15] E. Helpman, M.J. Melitz, and Yeaple. Export versus FDI with heterogeneous firms. Amer. Econ. Rev., 94(1):300-316, 2004.

[16] I. Higueras and R. März. Differential algebraic equations with properly stated leading terms. Comp. Math. Appl., 48:215-235, 2004. MR2086798 (2005e:34003)

[17] I. Higueras, R. März, and C. Tischendorf. Stability preserving integration of index-1 DAEs. Appl. Numer. Math, 45:175-200, 2003. MR1967573(2004b:34008)

[18] I. Higueras, R. März, and C. Tischendorf. Stability preserving integration of index-2 DAEs. Appl. Numer. Math, 45:201-229, 2003. MR1967574(2004b:34009)

[19] F. B. Hildebrand. Introduction to Numerical Analysis. McGraw-Hill, New York, 2nd edition, 1974. MR0347033(49:11753)

[20] F.R. de Hoog and R. Weiss. Difference methods for boundary value problems with a singularity of the first kind. SIAM J. Numer. Anal., 13:775-813, 1976. MR0440931 (55:13799)

[21] F.R. de Hoog and R. Weiss. Collocation methods for singular boundary value problems. SIA M J. Numer. Anal., 15:198-217, 1978. MR0468203 (57:8041)

[22] F.R. de Hoog and R. Weiss. The application of Runge-Kutta schemes to singular initial value problems. Math. Comp., 44:93-103, 1985. MR771033(86h:65100)

[23] T. Kapitula. Existence and stability of singular heteroclinic orbits for the Ginzburg-Landau equation. Nonlinearity, 9:669-685, 1996. MR1393152 (97d:35208)

[24] B. Karabay. Foreign direct investment and host country policies: A rationale for using ownership restrictions. Technical report, University of Virginia, WP, 2005.

[25] O. Koch. Asymptotically correct error estimation for collocation methods applied to singular boundary value problems. Numer. Math., 101:143-164, 2005. MR2194722 (2006k:65189)

[26] O. Koch, P. Kofler, and E. Weinmüller. Initial value problems for systems of ordinary first and second order differential equations with a singularity of the first kind. Analysis, 21:373-389, 2001. MR 1867622 (2002j:34005)

[27] O. Koch, R. März, D. Praetorius, and E.B. Weinmüller. Collocation methods for index-1 DAEs with a critical point. Mathematisches Forschungszentrum Oberwolfach, Report No. 18:81-84, 2006.

[28] O. Koch, R. März, D. Praetorius, and E.B. Weinmüller. Collocation for solving DAEs with singularities. Institute for Analysis and Scientific Computing, Report 32, Vienna University of Technology, 2007.

[29] O. Koch and E. Weinmüller. The convergence of shooting methods for singular boundary value problems. Math. Comp., 72(241):289-305, 2003. MR1933822 (2003h:65093)

[30] O. Koch and E. Weinmüller. Analytical and numerical treatment of a singular initial value problem in avalanche modeling. Appl. Math. Comput., 148(2):561-570, 2004. MR2015391 (2004j:34012)

[31] A. Kopelmann. Ein Kollokationsverfahren für überführbare Algebro-Differentialgleichungen. Preprint 1987-151, Humboldt University Berlin, 1987.

[32] P. Kunkel and V. Mehrmann. Differential-Algebraic Equations-Analysis and Numerical Solution. EMS Publishing House, Zürich, Switzerland, 2006. MR2225970 (2007e:34001)

[33] P. Kunkel and R. Stöver. Symmetric collocation methods for linear differential-algebraic boundary value problems. Numer. Math., 91:475-501, 2002. MR 1907868(2003e:65137)

[34] R. März. Differential algebraic equations anew. Appl. Numer. Math., 42:315-335, 2002. MR 1921345 (2003f:34001)

[35] R. März. The index of linear differential algebraic equations with properly stated leading terms. Results Math., 42:308-338, 2002. MR.1946748 (2003i:34004)

[36] R. März and R. Riaza. Linear differential-algebraic equations with properly stated leading terms: A-critical points. Math. Comp. Model. Dyn. Sys., 13:291-314, 2004. MR2333507 (2008e:34006)

[37] D. M. McClung and A. I. Mears. Dry-flowing avalanche run-up and run-out. J. Glaciol., 41(138):359-369, 1995.

[38] G. Moore. Geometric methods for computing invariant manifolds. Appl. Numer. Math., 17:319-331, 1995. MR1355567 (96g:65082)

[39] V.V. Ranade. Computational flow modeling for chemical engineering. Academic Press, San Diego, 2002.

[40] R. Riaza. Differential-Algebraic Systems. Analytical Aspects and Circuit Applications. World Scientific, 2008. MR2426820 
[41] K. Sundmacher and U. Hoffmann. Multicomponent mass and energy transport on different length scales in a packed reactive distillation column for heterogeneously catalyzed fuel ether production. Chem. Eng. Sci., 49:4443-4464, 1994.

[42] Chin-Yu Yeh, A.-B. Chen, D.M. Nicholson, and W.H. Butler. Full-potential Korringa-KohnRostoker band theory applied to the Mathieu potential. Phys. Rev. B, 42(17):10976-10982, 1990.

Vienna University of Technology, Institute for Analysis and Scientific Computing, Wiedner Hauptstr. 8-10, A-1040 Wien, Austria

E-mail address: othmar@othmar-koch.org

Humboldt-Universität of Berlin, Institute for Mathematics, Unter den Linden 6, D-10099 Berlin, Germany

E-mail address: maerz@mathematik.hu-berlin.de

Vienna University of Technology, Institute for Analysis and Scientific Computing, Wiedner Hauptstr. 8-10, A-1040 Wien, Austria

E-mail address: dirk.praetorius@tuwien.ac.at

Vienna University of Technology, Institute for Analysis and Scientific Computing, Wiedner Hauptstr. 8-10, A-1040 Wien, Austria

E-mail address: ewa.weinmueller@tuwien.ac.at 